\title{
THERMODYNAMICALLY CONSISTENT SIMULATION OF NONISOTHERMAL DIFFUSE-INTERFACE TWO-PHASE FLOW WITH PENG-ROBINSON EQUATION OF STATE*
}

\author{
JISHENG $\mathrm{KOU}^{\dagger}$ AND SHUYU SUN
}

\begin{abstract}
In this paper, we consider a diffuse-interface gas-liquid two-phase flow model with inhomogeneous temperatures, in which we employ the Peng-Robinson equation of state and the temperature-dependent influence parameter instead of the van der Waals equation of state and the constant influence parameter used in the existing models. As a result, our model can characterize accurately the physical behaviors of numerous realistic gas-liquid fluids, especially hydrocarbons. Furthermore, we prove a relation associating the pressure gradient with the gradients of temperature and chemical potential, and thereby derive a new formulation of the momentum balance equation, which shows that gradients of the chemical potential and temperature become the primary driving force of the fluid motion. It is rigorously proved that the new formulations of the model obey the first and second laws of thermodynamics. To design efficient numerical methods, we prove that Helmholtz free energy density is a concave function with respect to the temperature under certain physical conditions. Based on the proposed modeling formulations and the convex-concave splitting of Helmholtz free energy density, we propose a novel thermodynamically stable numerical scheme. We rigorously prove that the proposed method satisfies the first and second laws of thermodynamics. Finally, numerical tests are carried out to verify the effectiveness of the proposed simulation method.
\end{abstract}

Key words. Diffuse-interface model; Nonisothermal flow; Gas-liquid flow; Thermodynamical consistency; Peng-Robinson equation of state; Convex-concave splitting.

AMS subject classifications. 65N12; 76T10; 49S05

1. Introduction. Modeling and simulation of gas-liquid two-phase flow has a wide range of applications in industrial and scientific problems. In the oil reservoir, hydrocarbon is usually split into gas and liquid phases due to the effect of temperature, pressure and gravity. In the thermal enhanced oil recovery [6], heat is introduced intentionally to reduce the oil viscosity or vaporize part of the oil for the purpose of decreasing the mobility such that oil flows more freely through the reservoir. The heated oil may also vaporize and then form better oil once it condenses. In the natural world, gas-liquid two-phase flow is also one of the commonest phenomena, such as boiling, evaporation, and condensation [19. In this paper, we mainly focus on the diffuse-interface model of the gas-liquid two-phase flow at the pore scale, but which has indeed general formulations and can be applied for other applications.

In order to describe a gas-liquid interface, van der Waals introduced a gradient term in the Helmholtz free energy density, see 22] and the references therein. Korteweg developed the so-called Korteweg stress formulation induced by composition gradients, see [19,22 and the references therein. From then on, the diffuseinterface models for two-phase fluid flow have been extensively developed in the literature, 1, 3, 5, for instance.

In the traditional theories of phase transitions, the temperature is usually assumed to be constant. However, there exist many situations in which phase transitions are

${ }^{*}$ This work is supported by National Natural Science Foundation of China (No.11301163), and KAUST research fund to the Computational Transport Phenomena Laboratory at KAUST.

${ }^{\dagger}$ School of Mathematics and Statistics, Hubei Engineering University, Xiaogan 432000, Hubei, China.

${ }^{\ddagger}$ Corresponding author. Computational Transport Phenomena Laboratory, Division of Physical Science and Engineering, King Abdullah University of Science and Technology, Thuwal 23955-6900, Kingdom of Saudi Arabia. Email: shuyu.sun@kaust.edu.sa. 
strongly influenced by an inhomogeneous temperature field, such as boiling, evaporation, condensation and thermal enhanced oil recovery. To deal with such problems, a diffuse-interface model accounting for variable temperatures has been developed in 21,22] based on thermodynamical relations, and recently, [19] proposed a continuum mechanics modeling framework for liquid-vapor flows using the thermodynamical laws. Such models have been applied and extended to investigate the fluid problems with inhomogeneous temperatures [4,28, for instance. In such models, the Helmholtz free energy density usually consists of two contributions: one results from the bulk phase of a fluid, which can be formulated by van der Waals equation of state, and the other is the density gradient contribution on the two-phase interfaces. Although the van der Waals equation of state is popularly used in physics, the Peng-Robinson equation of state [23] has more accuracy for numerous realistic gas-liquid fluids including $\mathrm{N}_{2}, \mathrm{CO}_{2}$, and hydrocarbons; as a result, it has been extensively employed in petroleum and chemical industries. In recent years, modeling and simulation of two-phase flow based on the Peng-Robinson equation of state have become an attractive and challenging research topic in the reservoir and chemical engineering [10, 12, 17, 25, 27. In this paper, we will study a nonisothermal diffuse-interface model combining with the Peng-Robinson equation of state. In practices, the existing models usually employ a constant influence parameter in the density gradient contribution of Helmholtz free energy density, even though [22] assumed that such parameter can depend on the density. However, this influence parameter is generally viewed to highly depend on the temperature [20. Here, we will adapt a realistic formulation of the influence parameter, which is a function of temperature being consistent with the Peng-Robinson equation of state.

The models of [19,21,22] use a thermodynamic pressure, which is a function of the molar density and temperature. However, the pressure has a complicate formulation, which causes inconvenience in theoretical analysis and construction of numerical methods. In this paper, we will investigate a relation between the gradients of pressure, temperature and chemical potential, and from this, we can simplify the modeling equations, which allow us to conveniently prove the satisfaction of thermodynamical laws and to design efficient numerical schemes.

For numerical simulation of diffuse-interface models, it is demanded that numerical schemes shall satisfy the laws of thermodynamics due to the physical processes obeying such laws. More precisely speaking, for the motion of a fluid with inhomogeneous temperatures, the first law of thermodynamics (i.e. the energy balance law) is a basic physical principle, and once it is satisfied, we may obtain reliable and accurate results from numerical simulations. The second law of thermodynamics describes the entropy production of the realistic irreversible processes. As shown in [18, for a system under a fixed temperature, one can derive a formulation of entropy by the first law of thermodynamics, and then from the second law of thermodynamics, one can further derive the total (free) energy dissipation law, which is admitted in the phase-field model 3.7.26. Thus, a main challenge in numerical simulation is to design efficient numerical schemes that still satisfy the laws of thermodynamics. However, there are too few such methods available in the literature due to the short development history and more complications of these problems. A notable progress is that a provably entropy-stable numerical scheme was designed and analyzed in [19, which is based fundamentally on the concept of functional entropy variables.

It is different from the numerical schemes developed in 19 that our proposed numerical schemes will be designed using the convex-concave splitting of Helmholtz 
free energy density. For phase-field models, there are a lot of efforts on the developments of energy-dissipated schemes in the literature, [7,26] for instance, in which the convex-concave splitting of free energy functions is a key and efficient technique. For the Peng-Robinson equation of state, [25] analyzed the convex-concave splitting of the Helmholtz free energy density with respect to molar density, but its convex-concave property with respect to the temperature (which is a key point for the problem considered in this work) is not explored yet.

Another challenge in the considered modeling equations is the strongly nonlinear, tightly mutual coupling relationship between molar density, temperature and velocity. The resulted discrete equations in [19] is still strongly nonlinear and fully coupled. The approach of constructing an auxiliary velocity can be used to reduce the tight coupling relation between the phase function and velocity in phase filed simulation 26. In this paper, we extend this approach to the considered problem, and we define an auxiliary velocity, which depends on molar density and temperature. As a result, the nonlinear coupling relation in the proposed numerical scheme is alleviated to a great extent. We further propose a decoupled, linearized iterative method for solving the discrete equations, which satisfies the discrete first law of thermodynamics.

The key contributions of our work are listed as below:

(1) The Peng-Robinson equation of state is employed to replace the van der Waals equation of state in the existing nonisothermal diffuse-interface two-phase flow models. Moreover, we use a realistic formulation for the influence parameter in the gradient contribution of Helmholtz free energy density, which is a function of temperature instead of taking a constant as in the existing models. As a result, this modified model can characterize accurately the physical behaviors of numerous realistic gasliquid fluids including $\mathrm{N}_{2}, \mathrm{CO}_{2}$ and hydrocarbons etc.

(2) A relation associating the pressure gradient with the gradients of temperature and chemical potential is proved, and from this, we propose a new formulation of the momentum balance equation, which demonstrates that chemical potential and temperature gradients become the primary driving force of the fluid motion. The energy balance equation is also simplified. With the new formulations, it is convenient to prove that the model obeys the first and second laws of thermodynamics.

(3) We analyze the convex-concave splitting of Helmholtz free energy density; in particular, we prove that its bulk contribution is a concave function with respect to the temperature, and show that its gradient contribution is concave with respect to the temperature under certain conditions.

(4) Based on the proposed modeling formulations, combining the convex-concave splitting of Helmholtz free energy density, we propose a novel thermodynamically consistent numerical scheme, in which an auxiliary velocity is introduced to treat the coupling relations between molar density, velocity and temperature. We prove that the proposed method rigorously satisfies the first and second laws of thermodynamics.

Here, we note that thermodynamical consistency of a model or a numerical method means that such model or such method obeys the first and second laws of thermodynamics. Thermodynamical consistency is also called as thermodynamical stability.

The body of this paper is organized as follows. In Section 2, we will introduce the thermodynamic formulations derived from Peng-Robinson equation of state, the temperature-dependent influence parameter, and modeling equations of two-phase diffuse-interface flow with a variable temperature field. In Section 3, the modeling equations are simplified with the help of a relation between the gradients of pressure, 
temperature and chemical potential; subsequently, it is proved that the simplified model obeys the laws of thermodynamics. In Section 4, we propose a thermodynamically consistent numerical method based on an auxiliary velocity and the convexconcave splitting of Helmholtz free energy density, and we also prove that the proposed scheme satisfies the discrete laws of thermodynamics. In Section 5, numerical tests are carried out to verify effectiveness of the proposed method. Finally, some concluding remarks are provided in Section 6.

2. Mathematical model. In this section, we first describe the expressions of the thermodynamical variables and temperature-dependent influence parameter, and subsequently we formulate the modeling equations of a diffuse-interface two-phase flow model with a variable temperature field.

2.1. Formulations of thermodynamical variables. We consider a pure substance fluid, and let $n$ to denote the molar density of the substance. We now present the formulations of Helmholtz free energy density, entropy and internal energy, which are derived from Peng-Robinson equation of state [23, 27]. Let $T$ be the absolute temperature. We denote by $T_{c}$ and $P_{c}$ the critical temperature and critical pressure, respectively, and let the reduced temperature be $T_{r}=T / T_{c}$. Let $a$ and $b$ be the energy parameter and the covolume, respectively, which are calculated as

$$
a(T)=0.45724 \frac{R^{2} T_{c}^{2}}{P_{c}}\left[1+m\left(1-\sqrt{T_{r}}\right)\right]^{2}, \quad b=0.07780 \frac{R T_{c}}{P_{c}},
$$

where $R$ is the ideal gas constant. The coefficient $m$ is calculated by the following formulas

$$
\begin{gathered}
m=0.37464+1.54226 \omega-0.26992 \omega^{2}, \omega \leq 0.49, \\
m=0.379642+1.485030 \omega-0.164423 \omega^{2}+0.016666 \omega^{3}, \omega>0.49,
\end{gathered}
$$

where $\omega$ is the acentric factor.

The correlation coefficients $\alpha_{i}$ estimate the molar heat capacity of ideal gas at the constant pressure as [27]

$$
\psi_{p}(T)=\sum_{i=0}^{3} \alpha_{i} T^{i} .
$$

The bulk Helmholtz free energy density, denoted by $f_{b}$, is calculated as a sum of three contributions

$$
f_{b}(n, T)=f_{b}^{\text {ideal }}(n, T)+f_{b}^{\text {repulsion }}(n, T)+f_{b}^{\text {attraction }}(n, T),
$$

where

$$
\begin{gathered}
f_{b}^{\text {ideal }}(n, T)=n \vartheta_{0}+n \sum_{i=0}^{3} \alpha_{i} \frac{T^{i+1}-T_{0}^{i+1}}{i+1}-n R\left(T-T_{0}\right) \\
-n R T \ln \left(\frac{P_{0}}{n R T}\right)-n T \int_{T_{0}}^{T} \frac{\psi_{p}(\xi)}{\xi} d \xi \\
f_{b}^{\text {repulsion }}(n, T)=-n R T \ln (1-b n)
\end{gathered}
$$




$$
f_{b}^{\text {attraction }}(n, T)=\frac{a(T) n}{2 \sqrt{2} b} \ln \left(\frac{1+(1-\sqrt{2}) b n}{1+(1+\sqrt{2}) b n}\right),
$$

where $T_{0}=298.15 \mathrm{~K}, P_{0}=1 \mathrm{bar}$, and $\vartheta_{0}=-2478.95687512 \mathrm{~J} / \mathrm{mol}$. We note that the ideal contribution $f_{b}^{\text {ideal }}$ is indeed enriched by the heat capacity term.

The bulk internal energy, denoted by $\vartheta_{b}$, is formulated as [27]

$$
\begin{aligned}
\vartheta_{b}(n, T)= & n \vartheta_{0}+n \sum_{i=0}^{3} \alpha_{i} \frac{T^{i+1}-T_{0}^{i+1}}{i+1}-n R\left(T-T_{0}\right) \\
& +\frac{n\left(a(T)-T a^{\prime}(T)\right)}{2 \sqrt{2} b} \ln \left(\frac{1+(1-\sqrt{2}) b n}{1+(1+\sqrt{2}) b n}\right),
\end{aligned}
$$

where $a^{\prime}(T)$ denotes the derivative with respect to $T$. We denote by $s_{b}$ the bulk entropy and express it as 27

$$
\begin{aligned}
s_{b}(n, T)= & n R \ln (1-b n)+n R \ln \left(\frac{P_{0}}{n R T}\right)+n \int_{T_{0}}^{T} \frac{\psi_{p}(\xi)}{\xi} d \xi \\
& -\frac{n a^{\prime}(T)}{2 \sqrt{2} b} \ln \left(\frac{1+(1-\sqrt{2}) b n}{1+(1+\sqrt{2}) b n}\right) .
\end{aligned}
$$

The influence parameter generally relies on the temperature but independent of the molar density. We denote the influence parameters by $c$, which is given by [20]

$$
c(T)=a(T) b^{2 / 3}\left[\beta_{1}\left(1-T_{r}\right)+\beta_{2}\right],
$$

where $a$ and $b$ are the energy parameter and the covolume respectively and the coefficients $\beta_{1}$ and $\beta_{2}$ are calculated as

$$
\beta_{1}=-\frac{10^{-16}}{1.2326+1.3757 \omega}, \quad \beta_{2}=\frac{10^{-16}}{0.9051+1.5410 \omega} .
$$

We now express the density gradient contribution to Helmholtz free energy density and denote it by $f_{\nabla}$ :

$$
f_{\nabla}=\frac{1}{2} c \nabla n \cdot \nabla n
$$

The general Helmholtz free energy density (denoted by $f$ ) is a sum of two contributions:

$$
f=f_{b}+f_{\nabla}
$$

By thermodynamical relations [ 8 , the entropy density (denoted by $s$ ) and chemical potential (denoted by $\mu$ ) can be expressed as

$$
s=-\left(\frac{\delta f(n, T)}{\delta T}\right)_{n}, \quad \mu=\left(\frac{\delta f(n, T)}{\delta n}\right)_{T},
$$

where $\frac{\delta f}{\delta T}$ and $\frac{\delta f}{\delta n}$ represent the variational derivatives. We further define

$$
\gamma=\left(\frac{\delta f(n, T)}{\delta T}\right)_{n}=\gamma_{b}+\gamma_{\nabla}
$$


where $\gamma_{b}=\left(\frac{\partial f_{b}(n, T)}{\partial T}\right)_{n}$ and

$$
\gamma_{\nabla}=\left(\frac{\delta f_{\nabla}}{\delta T}\right)_{n}=\frac{1}{2} c^{\prime}(T)|\nabla n|^{2} .
$$

We denote the entropy contribution of the bulk fluid by $s_{b}$ and the entropy gradient contribution by $s_{\nabla}$. The thermodynamical relation yields

$$
s=s_{b}+s_{\nabla}=-\gamma_{b}-\gamma_{\nabla} .
$$

Let the bulk chemical potential be $\mu_{b}=\left(\frac{\partial f_{b}(n, T)}{\partial n}\right)_{T}$. The general form of chemical potential is expressed as

$$
\mu=\left(\frac{\delta f(n, T)}{\delta n}\right)_{T}=\mu_{b}+\mu_{\nabla}
$$

where $\mu_{\nabla}$ is the gradient contribution of chemical potential

$$
\mu_{\nabla}=\left(\frac{\delta f_{\nabla}(n, T)}{\delta n}\right)_{T}=-\nabla \cdot c \nabla n .
$$

2.2. Model equations. We now describe the modeling equations based on the models of [19,21,22], but the original van der Waals equation of state is replaced by the Peng-Robinson equation of state. Moreover, the temperature-dependent influence parameter given in (2.2) is adopt instead of constant parameters.

We denote the mass density by $\rho$ as $\rho=n M_{w}$, where $M_{w}$ is the molar weight. The fluid velocity is denoted by $\mathbf{u}$. The law of mass conservation states

$$
\frac{\partial n}{\partial t}+\nabla \cdot(n \mathbf{u})=0,
$$

which is also reformulated by a mass form

$$
\frac{\partial \rho}{\partial t}+\nabla \cdot(\rho \mathbf{u})=0 .
$$

The momentum balance equation is expressed as

$$
\frac{\partial(\rho \mathbf{u})}{\partial t}+\nabla \cdot(\rho \mathbf{u} \otimes \mathbf{u})=-\nabla \cdot \boldsymbol{\sigma},
$$

where $\boldsymbol{\sigma}$ is the total stress. Utilizing the mass conservation equation, we can reformulate (2.11) as

$$
\rho\left(\frac{\partial \mathbf{u}}{\partial t}+(\mathbf{u} \cdot \nabla) \mathbf{u}\right)=-\nabla \cdot \boldsymbol{\sigma} .
$$

For the realistic viscous flow, the total stress can be split into two parts: reversible part (denoted by $\boldsymbol{\sigma}_{\text {rev }}$ ) and irreversible part (denoted by $\boldsymbol{\sigma}_{\text {irrev }}$ ):

$$
\sigma=\sigma_{\text {rev }}+\sigma_{\text {irrev }} .
$$

The reversible stress has the form

$$
\boldsymbol{\sigma}_{\mathrm{rev}}=p \mathbf{I}+c(\nabla n \otimes \nabla n),
$$


where $p$ is the pressure and $\mathbf{I}$ is the second-order identity tensor. The pressure with density gradient contribution can be expressed as

$$
\begin{aligned}
p & =n \mu-f \\
& =n\left(\mu_{b}-\nabla \cdot c \nabla n\right)-f_{b}(n)-\frac{1}{2} c \nabla n \cdot \nabla n \\
& =p_{b}-n \nabla \cdot c \nabla n-\frac{1}{2} c \nabla n \cdot \nabla n,
\end{aligned}
$$

where $p_{b}$ is the bulk pressure as

$$
p_{b}=n \mu_{b}-f_{b} .
$$

Let $\eta$ and and $\xi$ represent the shear viscosity and volumetric viscosity respectively. We assume $\xi>\frac{2}{3} \eta$ as usual. Newtonian fluid theory suggests

$$
\boldsymbol{\sigma}_{\text {irrev }}=-\eta D(\mathbf{u})-(\lambda \nabla \cdot \mathbf{u}) \mathbf{I},
$$

where $D(\mathbf{u})=\nabla \mathbf{u}+\nabla \mathbf{u}^{T}$ and $\lambda=\xi-\frac{2}{3} \eta$.

We denote by $\vartheta$ the internal energy density per unit volume, and the total energy density includes the internal energy and kinetic energy as $e_{T}=\vartheta+\frac{1}{2} \rho|\mathbf{u}|^{2}$. The total energy balance equation is stated as

$$
\frac{\partial e_{T}}{\partial t}+\nabla \cdot\left(e_{T} \mathbf{u}+\boldsymbol{\sigma} \cdot \mathbf{u}\right)=\nabla \cdot(c(\nabla n \otimes \nabla n) \cdot \mathbf{u}-(\nabla \cdot(\mathbf{u} n)) c \nabla n)-\nabla \cdot \mathbf{q},
$$

where $\mathbf{q}$ is the heat transfer flux as

$$
\mathbf{q}=-\Theta \nabla T \text {. }
$$

Here, $\Theta$ denotes the heat diffusion coefficient, which depends generally on the molar density and temperature.

We now derive the equation of internal energy density from (2.17). Using the momentum balance equation, we obtain the transport of kinetic energy density as

$$
\begin{aligned}
\frac{1}{2} & \frac{\partial\left(\rho|\mathbf{u}|^{2}\right)}{\partial t}+\frac{1}{2} \nabla \cdot\left(\mathbf{u}\left(\rho|\mathbf{u}|^{2}\right)\right) \\
& =\rho \mathbf{u} \cdot \frac{\partial \mathbf{u}}{\partial t}+\frac{1}{2} \mathbf{u} \cdot \mathbf{u} \frac{\partial \rho}{\partial t}+\frac{1}{2}((\mathbf{u} \cdot \mathbf{u}) \nabla \cdot(\rho \mathbf{u})+2 \rho \mathbf{u} \cdot(\mathbf{u} \cdot \nabla \mathbf{u})) \\
& =\rho \mathbf{u} \cdot\left(\frac{\partial \mathbf{u}}{\partial t}+\mathbf{u} \cdot \nabla \mathbf{u}\right)+\frac{1}{2} \mathbf{u} \cdot \mathbf{u}\left(\frac{\partial \rho}{\partial t}+\nabla \cdot(\rho \mathbf{u})\right) \\
& =-\mathbf{u} \cdot \nabla \cdot \boldsymbol{\sigma} \\
& =-\nabla \cdot(\boldsymbol{\sigma} \cdot \mathbf{u})+\boldsymbol{\sigma}: \nabla \mathbf{u} .
\end{aligned}
$$

Substituting (2.19) into (2.17) yields the balance equation of internal energy density

$$
\frac{\partial \vartheta}{\partial t}+\nabla \cdot(\vartheta \mathbf{u})=\nabla \cdot(c(\nabla n \otimes \nabla n) \cdot \mathbf{u}-(\nabla \cdot(\mathbf{u} n)) c \nabla n)-\nabla \cdot \mathbf{q}-\boldsymbol{\sigma}: \nabla \mathbf{u} .(2
$$

We denote the bulk internal energy density by $\vartheta_{b}$. Then the thermodynamical relation gives

$$
\vartheta_{b}=f_{b}+s_{b} T .
$$

Furthermore, we denote by $\vartheta_{\nabla}$ the gradient contribution of internal energy density, and from the thermodynamical relation and formulations of $f_{\nabla}$ and $s_{\nabla}$, we obtain

$$
\vartheta_{\nabla}=f_{\nabla}+s_{\nabla} T=\frac{1}{2}\left(c(T)-T c^{\prime}(T)\right) \nabla n \cdot \nabla n .
$$


3. New formulations and thermodynamical consistency. As shown in the previous section, the reversible stress, consisting of the pressure and surface tension terms, has a complicate form. It is inconvenient for theoretical analysis and construction of numerical methods. In this section, we will prove a relation between the gradients of pressure, temperature and chemical potential, which allows us to simplify the momentum balance equation and energy balance equation. By the simplified equations, it is convenient to prove that the model obeys the first and second laws of thermodynamics.

3.1. New formulations. The following theorem provides a relation between the gradients of pressure, temperature and chemical potential.

THEOREM 3.1. The gradients of pressure, temperature and chemical potential have the following relation

$$
n \nabla \mu-\gamma \nabla T=\nabla p+\nabla \cdot c(\nabla n \otimes \nabla n) .
$$

Proof. First, we have the identity

$$
n \nabla \mu_{b}=\nabla p_{b}+\gamma_{b} \nabla T
$$

which is deduced from the formulation of $p_{b}$ as

$$
\nabla p_{b}=\nabla\left(n \mu_{b}-f_{b}\right)=n \nabla \mu_{b}+\mu_{b} \nabla n-\mu_{b} \nabla n-\gamma_{b} \nabla T=n \nabla \mu_{b}-\gamma_{b} \nabla T .
$$

From formulations of the pressure and chemical potential, taking into account (3.2), we derive the relation (3.1) as

$$
\begin{aligned}
n \nabla \mu-\nabla p & =n \nabla\left(\mu_{b}-\nabla \cdot(c \nabla n)\right)-\nabla\left(p_{b}-n \nabla \cdot(c \nabla n)-\frac{1}{2} c \nabla n \cdot \nabla n\right) \\
& =n \nabla \mu_{b}-\nabla p_{b}-n \nabla(\nabla \cdot(c \nabla n))+\nabla(n \nabla \cdot(c \nabla n))+\frac{1}{2} \nabla(c \nabla n \cdot \nabla n) \\
& =\gamma_{b} \nabla T+(\nabla \cdot(c \nabla n)) \nabla n+\frac{1}{2} \nabla(c \nabla n \cdot \nabla n) \\
& =\gamma_{b} \nabla T+(\nabla \cdot(c \nabla n)) \nabla n+\frac{1}{2}|\nabla n|^{2} \nabla c+\frac{1}{2} c \nabla|\nabla n|^{2} \\
& =\gamma_{b} \nabla T+\gamma_{\nabla} \nabla T+\nabla \cdot c(\nabla n \otimes \nabla n) \\
& =\gamma \nabla T+\nabla \cdot c(\nabla n \otimes \nabla n)
\end{aligned}
$$

where we have also used the identity

$$
(\nabla \cdot(c \nabla n)) \nabla n+\frac{1}{2} c \nabla|\nabla n|^{2}=\nabla \cdot c(\nabla n \otimes \nabla n) .
$$

This ends the proof. $\square$

Applying (3.1) to (2.12), we can obtain a new formulation of the momentum balance equation

$$
\rho\left(\frac{\partial \mathbf{u}}{\partial t}+(\mathbf{u} \cdot \nabla) \mathbf{u}\right)=-n \nabla \mu+\gamma \nabla T+\nabla \cdot \eta D(\mathbf{u})+\nabla(\lambda \nabla \cdot \mathbf{u}),
$$

which demonstrates that the gradients of chemical potential and temperature are the primal driving force. 
We now turn to simplify the energy balance equation. Applying (3.1) to (2.20), we derive

$$
\begin{aligned}
\frac{\partial \vartheta}{\partial t}+\nabla \cdot(\vartheta \mathbf{u})= & -\nabla \cdot(\mathbf{q}-c(\nabla n \otimes \nabla n) \cdot \mathbf{u}+(\nabla \cdot(\mathbf{u} n)) c \nabla n) \\
& -p \nabla \cdot \mathbf{u}-(c \nabla n \otimes \nabla n): \nabla \mathbf{u}-\boldsymbol{\sigma}_{\text {irrev }}: \nabla \mathbf{u} \\
= & -\nabla \cdot(\mathbf{q}+\mathbf{u} p+(\nabla \cdot(\mathbf{u} n)) c \nabla n) \\
& +\mathbf{u} \cdot \nabla p+\mathbf{u} \cdot \nabla \cdot(c \nabla n \otimes \nabla n)-\boldsymbol{\sigma}_{\text {irrev }}: \nabla \mathbf{u} \\
= & -\nabla \cdot(\mathbf{q}+\mathbf{u} p+(\nabla \cdot(\mathbf{u} n)) c \nabla n) \\
& +\mathbf{u} \cdot(n \nabla \mu-\gamma \nabla T)-\boldsymbol{\sigma}_{\text {irrev }}: \nabla \mathbf{u} \\
= & -\nabla \cdot(\mathbf{q}+(\nabla \cdot(\mathbf{u} n)) c \nabla n)+\nabla \cdot(\mathbf{u} f) \\
& -\mu \nabla \cdot(\mathbf{u} n)-\mathbf{u} \cdot \gamma \nabla T-\boldsymbol{\sigma}_{\text {irrev }}: \nabla \mathbf{u} .
\end{aligned}
$$

Moving the term $\nabla \cdot(\mathbf{u} f)$ into the left-hand side and taking into account $\vartheta=f+T s$, we obtain the balance equation of internal energy density

$$
\begin{aligned}
\frac{\partial \vartheta}{\partial t}+\nabla \cdot(s T \mathbf{u})= & -\nabla \cdot(\mathbf{q}+(\nabla \cdot(\mathbf{u} n)) c \nabla n) \\
& -\mu \nabla \cdot(\mathbf{u} n)-\mathbf{u} \cdot \gamma \nabla T-\boldsymbol{\sigma}_{\text {irrev }}: \nabla \mathbf{u} .
\end{aligned}
$$

We consider the fluids in a closed domain $\Omega$ with a fixed volume. The natural boundary conditions can be formulated as

$$
\mathbf{u}=0, \quad \nabla n \cdot \boldsymbol{\nu}_{\partial \Omega}=0 .
$$

where $\boldsymbol{\nu}_{\partial \Omega}$ denotes a normal unit outward vector to the boundary $\partial \Omega$. For the temperature, we partition the domain boundary $\partial \Omega$ into two non-overlapping subdivisions as $\partial \Omega=\Gamma_{n} \cup \Gamma_{d}$, and impose the boundary conditions

$$
\mathbf{q} \cdot \boldsymbol{\nu}_{\partial \Omega}=\mathbf{q}_{B} \text { on } \Gamma_{n}, \quad \text { and } \quad T=T_{B} \text { on } \Gamma_{d},
$$

where $\mathbf{q}_{B}$ is the given heat transfer flux across the boundary and $T_{B}$ is the given temperature distribution on the boundary. It is noted that either $\Gamma_{n}$ or $\Gamma_{d}$ may vanish or be redivided in a specific problem. The initial conditions for molar density, temperature and velocity are also provided.

In summary, the system of simplified modeling equations is composed of mass balance equation (2.9), the momentum balance equation (3.5), and the balance equation of internal energy density (3.7), as well as the initial and boundary conditions. As we will see in the next subsection, it is convenient to verify that this system satisfies the laws of thermodynamics.

3.2. Thermodynamical consistency. We first prove that the simplified model satisfies the first law of thermodynamics. We define the kinetic energy and internal energy over the domain as

$$
\mathcal{H}=\frac{1}{2} \int_{\Omega} \rho|\mathbf{u}|^{2} d \mathbf{x}, \quad \mathcal{U}=\int_{\Omega} \vartheta d \mathbf{x} .
$$

Furthermore, we define the total energy $\mathcal{E}$ over the domain

$$
\mathcal{E}=\int_{\Omega} e_{T} d \mathbf{x}=\mathcal{H}+\mathcal{U}
$$


TheOrem 3.2. The system of equations (2.9), (3.5) and (3.7) satisfies the first law of thermodynamics as

$$
\frac{\partial \mathcal{E}}{\partial t}=-\int_{\partial \Omega} \mathbf{q} \partial \Omega \cdot \boldsymbol{\nu}_{\partial \Omega} d \boldsymbol{s}
$$

where $\mathbf{q} \partial \Omega$ denotes the heat transfer flux between the system and its environment and $\nu_{\partial \Omega}$ denotes a normal unit outward vector to the boundary $\partial \Omega$.

Proof. From (2.19), we get

$$
\frac{\partial \mathcal{H}}{\partial t}=\int_{\Omega} \rho \mathbf{u} \cdot\left(\frac{\partial \mathbf{u}}{\partial t}+\mathbf{u} \cdot \nabla \mathbf{u}\right) d \mathbf{x}
$$

Substituting (3.5) into (3.12) yields

$$
\begin{aligned}
\frac{\partial \mathcal{H}}{\partial t} & =\int_{\Omega} \mathbf{u} \cdot(-n \nabla \mu+\gamma \nabla T+\nabla \cdot \eta D(\mathbf{u})+\nabla \lambda \nabla \cdot \mathbf{u}) d \mathbf{x} \\
& =\int_{\Omega} \mathbf{u} \cdot(\gamma \nabla T-n \nabla \mu) d \mathbf{x}+\int_{\Omega} \boldsymbol{\sigma}_{\text {irrev }}: \nabla \mathbf{u} d \mathbf{x} .
\end{aligned}
$$

Integrating (3.7) over $\Omega$ yields

$$
\frac{\partial \mathcal{U}}{\partial t}=-\int_{\partial \Omega} \mathbf{q} \partial \Omega \cdot \boldsymbol{\nu}_{\partial \Omega} d s-\int_{\Omega}\left(\mu \nabla \cdot(\mathbf{u} n)+\mathbf{u} \cdot \gamma \nabla T+\boldsymbol{\sigma}_{\text {irrev }}: \nabla \mathbf{u}\right) d \mathbf{x} .
$$

Taking a summation of (3.13) and (3.14), and then taking into account

$$
\int_{\Omega}(\mu \nabla \cdot(\mathbf{u} n)+n \mathbf{u} \cdot \nabla \mu) d \mathbf{x}=\int_{\Omega} \nabla \cdot(\mu \mathbf{u} n) d \mathbf{x}=0,
$$

we obtain (3.11).

We next prove that the simplified model system satisfies the second law of thermodynamics. For the notations, we use $(\cdot, \cdot)$ and $\|\cdot\|$ to represent the $L^{2}(\Omega),\left(L^{2}(\Omega)\right)^{d}$ or $\left(L^{2}(\Omega)\right)^{d \times d}$ inner product and norm respectively. We define the entropy over the domain and denote it by $\mathcal{S}$. Since $s=\frac{1}{T}(\vartheta-f)$, we obtain

$$
\frac{\partial \mathcal{S}}{\partial t}=\left(\frac{\partial s}{\partial t}, 1\right)=\left(\frac{\partial(\vartheta-f)}{\partial t}, \frac{1}{T}\right)-\left(\frac{\partial T}{\partial t}, \frac{s}{T}\right) .
$$

In order to estimate the entropy, we first need to derive a variation equation of Helmholtz free energy density.

Lemma 3.1. The Helmholtz free energy density satisfies the following variation equation

$$
\frac{\partial f}{\partial t}=\gamma \frac{\partial T}{\partial t}-\mu \nabla \cdot(n \mathbf{u})-\nabla \cdot((\nabla \cdot(\mathbf{u} n)) c \nabla n)
$$

Proof. First, using the mass conservation equation, we obtain the variation of the bulk Helmholtz free energy $f_{b}$ as

$$
\frac{\partial f_{b}}{\partial t}=\mu_{b} \frac{\partial n}{\partial t}+\gamma_{b} \frac{\partial T}{\partial t}
$$




$$
=-\mu_{b} \nabla \cdot(n \mathbf{u})+\gamma_{b} \frac{\partial T}{\partial t} .
$$

Furthermore, we derive the variation of the gradient contribution of Helmholtz free energy $f_{\nabla}$ as

$$
\begin{aligned}
\frac{\partial f_{\nabla}}{\partial t} & =\frac{1}{2} \frac{\partial(c \nabla n \cdot \nabla n)}{\partial t} \\
& =\frac{1}{2}|\nabla n|^{2} \frac{\partial c}{\partial t}+\frac{1}{2} c \frac{\partial(\nabla n \cdot \nabla n)}{\partial t} \\
& =\gamma_{\nabla} \frac{\partial T}{\partial t}+c \nabla n \cdot \nabla \frac{\partial n}{\partial t} \\
& =\gamma_{\nabla} \frac{\partial T}{\partial t}-c \nabla n \cdot \nabla(\nabla \cdot(\mathbf{u} n)) \\
& =\gamma_{\nabla} \frac{\partial T}{\partial t}-\nabla \cdot((\nabla \cdot(\mathbf{u} n)) c \nabla n)-\mu_{\nabla} \nabla \cdot(\mathbf{u} n) .
\end{aligned}
$$

Thus, (3.16) is obtained summing (3.17) and (3.18). $\mathrm{C}$

THEOREM 3.3. The system of equations (2.9), (3.5) and (3.7) satisfies the second law of thermodynamics as

$$
\begin{aligned}
\frac{\partial \mathcal{S}}{\partial t}+\int_{\partial \Omega} \frac{\mathbf{q} \partial \Omega \cdot \boldsymbol{\nu}_{\partial \Omega}}{T} d s= & \left\|\frac{\Theta^{1 / 2}}{T} \nabla T\right\|^{2}+\frac{1}{2}\left\|\left(\frac{\eta}{T}\right)^{1 / 2} D(\mathbf{u})\right\|^{2} \\
& +\left\|\left(\frac{\lambda}{T}\right)^{1 / 2} \nabla \cdot \mathbf{u}\right\|^{2}
\end{aligned}
$$

where $\mathbf{q} \partial \Omega$ denotes the heat transfer flux between the system and its environment and $\boldsymbol{\nu}_{\partial \Omega}$ denotes a normal unit outward vector to the boundary $\partial \Omega$.

Proof. We combine (3.7) and (3.16) and obtain

$$
\frac{\partial(\vartheta-f)}{\partial t}+\nabla \cdot(s T \mathbf{u})=-\nabla \cdot \mathbf{q}-\mathbf{u} \cdot \gamma \nabla T-\boldsymbol{\sigma}_{\mathrm{irrev}}: \nabla \mathbf{u}-\gamma \frac{\partial T}{\partial t} .
$$

Since

$$
\nabla \cdot(s T \mathbf{u})=T \nabla \cdot(s \mathbf{u})+s \mathbf{u} \cdot \nabla T=T \nabla \cdot(s \mathbf{u})-\gamma \mathbf{u} \cdot \nabla T,
$$

the equation (3.20) can be reduced into

$$
\frac{\partial(\vartheta-f)}{\partial t}=-T \nabla \cdot(s \mathbf{u})-\nabla \cdot \mathbf{q}-\boldsymbol{\sigma}_{\text {irrev }}: \nabla \mathbf{u}-\gamma \frac{\partial T}{\partial t} .
$$

Substituting (3.21) into (3.15), and taking into account $s=-\gamma$, we derive the entropy variation with time

$$
\begin{aligned}
\frac{\partial \mathcal{S}}{\partial t} & =-(\nabla \cdot(s \mathbf{u}), 1)+\left(\nabla \cdot \Theta \nabla T, \frac{1}{T}\right)-\left(\boldsymbol{\sigma}_{\text {irrev }}: \nabla \mathbf{u}, \frac{1}{T}\right) \\
& =-\int_{\partial \Omega} \frac{\mathbf{q} \partial \Omega \cdot \boldsymbol{\nu}_{\partial \Omega}}{T} d s+\left\|\frac{\Theta^{1 / 2}}{T} \nabla T\right\|^{2}-\left(\boldsymbol{\sigma}_{\text {irrev }}: \nabla \mathbf{u}, \frac{1}{T}\right) .
\end{aligned}
$$

Applying the formulation of $\boldsymbol{\sigma}_{\text {irrev }}$ to (3.22), we obtain the equation (3.19). 
4. Thermodynamically consistent numerical method. In this section, we focus on designing semi-implicit time marching schemes, which are based on the above simplified formulations and obey the laws of thermodynamics. For this purpose, it is a key ingredient to construct the convex-concave splitting of Helmholtz free energy density with respect to molar density and temperature. The other challenge results from the tight nonlinear coupling relation among molar density, temperature and velocity. In order to alleviate this relation, we will introduce an auxiliary velocity, which depends on molar density and temperature. Very careful physical observations are also required to treat this coupling relation by a way of semi-implicit time discretization.

We first consider the convex-concave splitting of bulk Helmholtz free energy density. It is noted that $\psi_{p}$ in the formulation of $f_{b}$ is the molar heat capacity of the ideal gas at the constant pressure. We recall the following thermodynamical relation for the ideal gas

$$
\psi_{p}=R+\psi_{v},
$$

where $R$ is the universal gas constant and $\psi_{v}>0$ is the molar heat capacity at the constant volume for the ideal gas.

Lemma 4.1. The bulk Helmholtz free energy density $f_{b}$ can be split into two parts: one is a convex function with respect to $n$, denoted by $f_{b}^{\operatorname{convex}}(n, T)$, and the other is a concave function with respect to $n$, denoted by $f_{b}^{\text {concave }}(n, T)$, which are formulated as

$$
\begin{gathered}
f_{b}^{\text {convex }}(n, T)=f_{b}^{\text {ideal }}(n, T)+f_{b}^{\text {repulsion }}(n, T), \\
f_{b}^{\text {concave }}(n, T)=f_{b}^{\text {attraction }}(n, T) .
\end{gathered}
$$

Moreover, $f_{b}$ is concave with respect to the temperature.

Proof. The convexity and concavity of $f_{b}$ with respect to molar density have been mostly proved in 25 although there exists a bit difference in the formulation of $f_{b}^{\text {ideal }}(n, T)$. We primarily prove the concave property of $f_{b}$ with respect to the temperature. The second derivative of $f_{b}$ with respect to $T$ can be calculated as

$$
\begin{aligned}
\frac{\partial^{2} f_{b}(n, T)}{\partial T^{2}} & =\frac{n R}{T}-n \frac{\psi_{p}(T)}{T}+\frac{n a^{\prime \prime}(T)}{2 \sqrt{2} b} \ln \left(\frac{1+(1-\sqrt{2}) b n}{1+(1+\sqrt{2}) b n}\right) \\
& =-n \frac{\psi_{v}(T)}{T}+\frac{n a^{\prime \prime}(T)}{2 \sqrt{2} b} \ln \left(\frac{1+(1-\sqrt{2}) b n}{1+(1+\sqrt{2}) b n}\right),
\end{aligned}
$$

where we have used the relation (4.1). From the definition of $a(T)$, we calculate

$$
\begin{gathered}
a^{\prime}(T)=-\frac{a(T)}{1+m\left(1-\sqrt{T_{r}}\right)} \frac{m}{\sqrt{T T_{c}}}, \\
a^{\prime \prime}(T)=\frac{m a(T)}{2 T \sqrt{T T_{c}}} \frac{1+m}{\left(1+m\left(1-\sqrt{T_{r}}\right)\right)^{2}} .
\end{gathered}
$$

We can see that $a^{\prime \prime}(T) \geq 0$, and then we conclude that $\frac{\partial^{2} f_{b}(n, T)}{\partial T^{2}} \leq 0$; that is, $f_{b}$ is concave with respect to the temperature.

Lemma 4.2. The gradient contribution to the Helmholtz free energy density is always convex with respect to molar density. Moreover, it is concave with respect to 
the temperature if we take the temperature such that

$$
(1+m)\left(\beta_{1}\left(1-T_{r}\right)+\beta_{2}\right)+4 \beta_{1} T_{r}\left(1+m\left(1-\sqrt{T_{r}}\right)\right) \leq 0,
$$

where $T_{r}=T / T_{c}$.

Proof. It is obvious that the gradient contribution to the Helmholtz free energy density is convex with respect to molar density. We now consider its concavity with respect to the temperature. The derivatives of $c(T)$ are calculated as

$$
\begin{gathered}
c^{\prime}(T)=a^{\prime}(T) b^{2 / 3}\left[\beta_{1}\left(1-T_{r}\right)+\beta_{2}\right]-a(T) b^{2 / 3} \frac{\beta_{1}}{T_{c}}, \\
c^{\prime \prime}(T)=a^{\prime \prime}(T) b^{2 / 3}\left[\beta_{1}\left(1-T_{r}\right)+\beta_{2}\right]-2 a^{\prime}(T) b^{2 / 3} \frac{\beta_{1}}{T_{c}} .
\end{gathered}
$$

Substituting $a^{\prime}(T)$ and $a^{\prime \prime}(T)$ into $c^{\prime \prime}(T)$, we obtain

$$
c^{\prime \prime}(T)=\frac{m a(T) b^{2 / 3}}{2 T \sqrt{T T_{c}}} \frac{(1+m)\left(\beta_{1}\left(1-T_{r}\right)+\beta_{2}\right)+4 \beta_{1} T_{r}\left(1+m\left(1-\sqrt{T_{r}}\right)\right)}{\left(1+m\left(1-\sqrt{T_{r}}\right)\right)^{2}} .
$$

which yields the concavity combining the condition (4.4). $\mathrm{Q}$

We make some remarks on the condition (4.4). In (4.4), the parameter $\beta_{1}$ has a negative value, while the rest parameters are positive, so the satisfaction of (4.4) is reasonable. We have checked in numerical tests that the condition (4.4) is satisfied for butane when the temperature lies in a large range from $0.1 T_{c}$ to $3 T_{c}$, where $T_{c}$ is the critical temperature of butane. So in what follows, we assume that the condition (4.4) always holds for our considered problems.

We now contruct the semi-implicit time marching scheme. A time interval $\mathcal{I}=$ $\left(0, T_{f}\right]$, where $T_{f}>0$, is considered, and we divide $\mathcal{I}$ into $M$ subintervals $\mathcal{I}_{k}=$ $\left(t_{k}, t_{k+1}\right]$, where $t_{0}=0$ and $t_{M}=T_{f}$. The time step size is denoted as $\delta t_{k}=$ $t_{k+1}-t_{k}$. For a scalar function $v(t)$ or a vector function $\mathbf{v}(t)$, we denote by $v^{k}$ or $\mathbf{v}^{k}$ its approximation at the time $t_{k}$. First, a semi-implicit time marching scheme accounting for the convex-splitting of Helmholtz free energy density is used to discretize the chemical potential

$$
\begin{aligned}
& \mu^{k+1}=\mu_{b}^{k+1}+\mu_{\nabla}\left(n^{k+1}, T^{k+1}\right), \\
& \mu_{b}^{k+1}=\mu_{b}^{\text {convex }}\left(n^{k+1}, T^{k+1}\right)+\mu_{b}^{\text {concave }}\left(n^{k}, T^{k+1}\right) .
\end{aligned}
$$

We define an auxiliary velocity as

$$
\mathbf{u}_{\star}^{k}=\mathbf{u}^{k}-\frac{\delta t_{k}}{\rho^{k}}\left(n^{k} \nabla \mu^{k+1}+s^{k} \nabla T^{k+1}\right),
$$

where $\rho^{k}=n^{k} M_{w}$. We take $\nabla \mu^{k+1} \cdot \boldsymbol{\nu}_{\partial \Omega}=\nabla T^{k+1} \cdot \boldsymbol{\nu}_{\partial \Omega}=0$ for $\mathbf{u}_{\star}^{k}$ on the boundary, and as a result, we have still $\mathbf{u}_{\star}^{k}=0$ on the boundary. $\mathbf{u}_{\star}^{k}$ can be viewed as an approximation of $\mathbf{u}^{k+1}$ obtained by neglecting the convection and viscosity terms in the momentum balance equation. Subsequently, a semi-implicit scheme is designed as below:

$$
\frac{n^{k+1}-n^{k}}{\delta t_{k}}+\nabla \cdot\left(n^{k} \mathbf{u}_{\star}^{k}\right)=0
$$




$$
\begin{aligned}
& \rho^{k}\left(\frac{\mathbf{u}^{k+1}-\mathbf{u}^{k}}{\delta t_{k}}+\mathbf{u}_{\star}^{k} \cdot \nabla \mathbf{u}^{k+1}\right)=-n^{k} \nabla \mu^{k+1}+\gamma^{k} \nabla T^{k+1} \\
& +\nabla \cdot \eta^{k} D\left(\mathbf{u}^{k+1}\right)+\nabla\left(\lambda^{k} \nabla \cdot \mathbf{u}^{k+1}\right), \\
& \frac{\vartheta^{k+1}-\vartheta^{k}}{\delta t_{k}}+\nabla \cdot\left(\mathbf{u}_{\star}^{k} s^{k} T^{k+1}\right)=-\nabla \cdot \mathbf{q}^{k+1} \\
& -\nabla \cdot\left(\left(\nabla \cdot\left(\mathbf{u}_{\star}^{k} n^{k}\right)\right) c^{k+1} \nabla n^{k+1}\right)-\mu^{k+1} \nabla \cdot\left(\mathbf{u}_{\star}^{k} n^{k}\right)-\mathbf{u}_{\star}^{k} \cdot \gamma^{k} \nabla T^{k+1} \\
& +\eta^{k} D\left(\mathbf{u}^{k+1}\right): \nabla \mathbf{u}^{k+1}+\lambda^{k}\left|\nabla \cdot \mathbf{u}^{k+1}\right|^{2} \\
& +\frac{1}{2 \delta t_{k}} \rho^{k}\left(\left|\mathbf{u}^{k+1}-\mathbf{u}_{\star}^{k}\right|^{2}+\left|\mathbf{u}_{\star}^{k}-\mathbf{u}^{k}\right|^{2}\right),
\end{aligned}
$$

where

$$
\mathbf{q}^{k+1}=-\Theta^{k} \nabla T^{k+1}, \quad \Theta^{k}=\Theta\left(n^{k}, T^{k}\right) .
$$

Using the identity

$$
\nabla \cdot\left(\mathbf{u}_{\star}^{k} s^{k} T^{k+1}\right)=T^{k+1} \nabla \cdot\left(\mathbf{u}_{\star}^{k} s^{k}\right)-\mathbf{u}_{\star}^{k} \cdot \gamma^{k} \nabla T^{k+1},
$$

we can also reformulate (4.9) as

$$
\begin{aligned}
& \frac{\vartheta^{k+1}-\vartheta^{k}}{\delta t_{k}}+T^{k+1} \nabla \cdot\left(\mathbf{u}_{\star}^{k} s^{k}\right)=-\nabla \cdot \mathbf{q}^{k+1} \\
& \quad-\nabla \cdot\left(\left(\nabla \cdot\left(\mathbf{u}_{\star}^{k} n^{k}\right)\right) c^{k+1} \nabla n^{k+1}\right)-\mu^{k+1} \nabla \cdot\left(\mathbf{u}_{\star}^{k} n^{k}\right) \\
& \quad+\eta^{k} D\left(\mathbf{u}^{k+1}\right): \nabla \mathbf{u}^{k+1}+\lambda^{k}\left|\nabla \cdot \mathbf{u}^{k+1}\right|^{2} \\
& \quad+\frac{1}{2 \delta t_{k}} \rho^{k}\left(\left|\mathbf{u}^{k+1}-\mathbf{u}_{\star}^{k}\right|^{2}+\left|\mathbf{u}_{\star}^{k}-\mathbf{u}^{k}\right|^{2}\right) .
\end{aligned}
$$

We now prove that the above semi-implicit scheme obeys the laws of thermodynamics. To do this, we define the discrete formulations of total energy, kinetic energy and internal energy over the domain at the time $t_{k}$ as

$$
\mathcal{E}^{k}=\mathcal{H}^{k}+\mathcal{U}^{k}, \quad \mathcal{H}^{k}=\frac{1}{2} \int_{\Omega} \rho^{k}\left|\mathbf{u}^{k}\right|^{2} d \mathbf{x}, \quad \mathcal{U}^{k}=\int_{\Omega} \vartheta^{k} d \mathbf{x} .
$$

THEOREM 4.1. The semi-implicit scheme given by (4.5)-(4.9) satisfies the first law of thermodynamics as

$$
\frac{\mathcal{E}^{k+1}-\mathcal{E}^{k}}{\delta t_{k}}=-\int_{\partial \Omega} \mathbf{q}_{\partial \Omega}^{k+1} \cdot \boldsymbol{\nu}_{\partial \Omega} d s
$$

where $\mathbf{q}_{\partial \Omega}^{k+1}$ denotes the heat transfer flux between the system and its environment at the time $t^{k+1}$.

Proof. Multiplying both sides of (4.8) by $\mathbf{u}^{k+1}$ and integrating it over $\Omega$, we obtain

$$
\begin{gathered}
\left(\rho^{k} \frac{\mathbf{u}^{k+1}-\mathbf{u}_{\star}^{k}}{\delta t_{k}}, \mathbf{u}^{k+1}\right)+\left(\rho^{k} \mathbf{u}_{\star}^{k} \cdot \nabla \mathbf{u}^{k+1}, \mathbf{u}^{k+1}\right) \\
=-\left\|\sqrt{\lambda^{k}} \nabla \cdot \mathbf{u}^{k+1}\right\|^{2}-\frac{1}{2}\left\|\sqrt{\eta^{k}} D\left(\mathbf{u}^{k+1}\right)\right\|^{2} .
\end{gathered}
$$


Using (4.7) and taking into account $\rho^{k}=n^{k} M_{w}$, we estimate

$$
\begin{aligned}
& \left(\rho^{k}\left(\mathbf{u}^{k+1}-\mathbf{u}_{\star}^{k}\right), \mathbf{u}^{k+1}\right)-\frac{1}{2}\left(\rho^{k},\left|\mathbf{u}^{k+1}-\mathbf{u}_{\star}^{k}\right|^{2}\right) \\
= & \frac{1}{2}\left(\rho^{k},\left|\mathbf{u}^{k+1}\right|^{2}-\left|\mathbf{u}_{\star}^{k}\right|^{2}\right) \\
= & \mathcal{H}^{k+1}-\mathcal{H}_{\star}^{k}-\frac{1}{2}\left(\rho^{k+1}-\rho^{k},\left|\mathbf{u}^{k+1}\right|^{2}\right) \\
= & \mathcal{H}^{k+1}-\mathcal{H}_{\star}^{k}+\frac{\delta t_{k}}{2}\left(\nabla \cdot\left(\rho^{k} \mathbf{u}_{\star}^{k}\right),\left|\mathbf{u}^{k+1}\right|^{2}\right) \\
= & \mathcal{H}^{k+1}-\mathcal{H}_{\star}^{k}-\delta t_{k}\left(\rho^{k} \mathbf{u}_{\star}^{k} \cdot \nabla \mathbf{u}^{k+1}, \mathbf{u}^{k+1}\right),
\end{aligned}
$$

where

$$
\mathcal{H}_{\star}^{k}=\frac{1}{2} \int_{\Omega} \rho^{k}\left|\mathbf{u}_{\star}^{k}\right|^{2} d \mathbf{x} .
$$

Substituting (4.13) into (4.12) yields

$$
\begin{aligned}
\frac{\mathcal{H}^{k+1}-\mathcal{H}_{\star}^{k}}{\delta t_{k}}= & -\frac{1}{2 \delta t_{k}}\left(\rho^{k},\left|\mathbf{u}^{k+1}-\mathbf{u}_{\star}^{k}\right|^{2}\right) \\
& -\left\|\sqrt{\lambda^{k}} \nabla \cdot \mathbf{u}^{k+1}\right\|^{2}-\frac{1}{2}\left\|\sqrt{\eta^{k}} D\left(\mathbf{u}^{k+1}\right)\right\|^{2} .
\end{aligned}
$$

We multiply both sides of (4.6) by $\mathbf{u}_{\star}^{k}$ and then integrate it over $\Omega$

$$
\begin{aligned}
\mathcal{H}_{\star}^{k}-\mathcal{H}^{k} & =\left(\rho^{k}\left(\mathbf{u}_{\star}^{k}-\mathbf{u}^{k}\right), \mathbf{u}_{\star}^{k}\right)-\frac{1}{2}\left(\rho^{k},\left|\mathbf{u}_{\star}^{k}-\mathbf{u}^{k}\right|^{2}\right) \\
& =-\delta t_{k}\left(n^{k} \nabla \mu^{k+1}+s^{k} \nabla T^{k+1}, \mathbf{u}_{\star}^{k}\right)-\frac{1}{2}\left(\rho^{k},\left|\mathbf{u}_{\star}^{k}-\mathbf{u}^{k}\right|^{2}\right) \\
& =\delta t_{k}\left(\left(\nabla \cdot\left(n^{k} \mathbf{u}_{\star}^{k}\right), \mu^{k+1}\right)-\left(s^{k} \nabla T^{k+1}, \mathbf{u}_{\star}^{k}\right)\right)-\frac{1}{2}\left(\rho^{k},\left|\mathbf{u}_{\star}^{k}-\mathbf{u}^{k}\right|^{2}\right)(
\end{aligned}
$$

Integrating (4.9) over $\Omega$ yields

$$
\begin{aligned}
& \frac{\mathcal{U}^{k+1}-\mathcal{U}^{k}}{\delta t_{k}}=-\int_{\partial \Omega} \mathbf{q}_{\partial \Omega}^{k+1} \cdot \boldsymbol{\nu}_{\partial \Omega} d s-\left(\mu^{k+1}, \nabla \cdot\left(\mathbf{u}_{\star}^{k} n^{k}\right)\right) \\
& -\left(\mathbf{u}_{\star}^{k}, \gamma^{k} \nabla T^{k+1}\right)+\left\|\sqrt{\lambda^{k}} \nabla \cdot \mathbf{u}^{k+1}\right\|^{2}+\frac{1}{2}\left\|\sqrt{\eta^{k}} D\left(\mathbf{u}^{k+1}\right)\right\|^{2} \\
& +\frac{1}{2 \delta t_{k}}\left(\rho^{k},\left|\mathbf{u}^{k+1}-\mathbf{u}_{\star}^{k}\right|^{2}+\left|\mathbf{u}_{\star}^{k}-\mathbf{u}^{k}\right|^{2}\right) .
\end{aligned}
$$

Summing (4.14), (4.15) and (4.16) yields (4.11).

We turn to prove that the proposed semi-implicit scheme obeys the second law of thermodynamics. We first need to prove a discrete analog of Lemma 3.1

Lemma 4.3. Assume that the condition (4.4) holds. The discrete Helmholtz free energy densities satisfy

$$
\begin{aligned}
\frac{f^{k+1}-f^{k}}{\delta t_{k}} \leq & \gamma^{k} \frac{T^{k+1}-T^{k}}{\delta t_{k}}-\mu^{k+1} \nabla \cdot\left(n^{k} \mathbf{u}_{\star}^{k}\right) \\
& -\nabla \cdot\left(\left(\nabla \cdot\left(\mathbf{u}_{\star}^{k} n^{k}\right)\right) c^{k+1} \nabla n^{k+1}\right),
\end{aligned}
$$

where $f^{k}=f\left(n^{k}, T^{k}\right)$. 
Proof. We utilize the properties of convex and concave functions to estimate the bulk Helmholtz free energy $f_{b}$ as

$$
\begin{aligned}
\frac{f_{b}^{k+1}-f_{b}^{k}}{\delta t_{k}} & =\frac{f_{b}\left(n^{k+1}, T^{k+1}\right)-f_{b}\left(n^{k}, T^{k+1}\right)}{\delta t_{k}}+\frac{f_{b}\left(n^{k}, T^{k+1}\right)-f_{b}\left(n^{k}, T^{k}\right)}{\delta t_{k}} \\
& \leq \mu_{b}^{k+1} \frac{n^{k+1}-n^{k}}{\delta t_{k}}+\gamma_{b}^{k} \frac{T^{k+1}-T^{k}}{\delta t_{k}} \\
& =-\mu_{b}^{k+1} \nabla \cdot\left(n^{k} \mathbf{u}_{\star}^{k}\right)+\gamma_{b}^{k} \frac{T^{k+1}-T^{k}}{\delta t_{k}}
\end{aligned}
$$

where we have also used the discrete equation of mass balance. Next, we consider the difference of the gradient contributions to Helmholtz free energy between two time steps as

$$
\begin{aligned}
f_{\nabla}^{k+1}-f_{\nabla}^{k} & =\frac{1}{2} c^{k+1} \nabla n^{k+1} \cdot \nabla n^{k+1}-\frac{1}{2} c^{k} \nabla n^{k} \cdot \nabla n^{k} \\
& =\frac{1}{2} c^{k+1}\left(\nabla n^{k+1} \cdot \nabla n^{k+1}-\nabla n^{k} \cdot \nabla n^{k}\right)+\frac{1}{2}\left(c^{k+1}-c^{k}\right) \nabla n^{k} \cdot \nabla n^{k} \\
& \leq \frac{1}{2} c^{k+1} \nabla\left(n^{k+1}+n^{k}\right) \cdot \nabla\left(n^{k+1}-n^{k}\right)+\gamma_{\nabla}^{k}\left(T^{k+1}-T^{k}\right),
\end{aligned}
$$

where the concavity of the function $c$ with respect to the temperature is used to get the last inequality. Using the discrete equation of mass balance and the definition of $\mu_{\nabla}^{k+1}$, we derive

$$
\begin{aligned}
& \frac{1}{2} c^{k+1} \nabla\left(n^{k+1}+n^{k}\right) \cdot \nabla\left(n^{k+1}-n^{k}\right) \\
& \quad=c^{k+1} \nabla n^{k+1} \cdot \nabla\left(n^{k+1}-n^{k}\right)-\frac{1}{2} c^{k+1} \nabla\left(n^{k+1}-n^{k}\right) \cdot \nabla\left(n^{k+1}-n^{k}\right) \\
& \quad \leq-\delta t_{k} c^{k+1} \nabla n^{k+1} \cdot \nabla\left(\nabla \cdot\left(\mathbf{u}_{\star}^{k} n^{k}\right)\right) \\
& \quad \leq-\delta t_{k} \nabla \cdot\left(\left(\nabla \cdot\left(\mathbf{u}_{\star}^{k} n^{k}\right)\right) c^{k+1} \nabla n^{k+1}\right)-\delta t_{k} \mu_{\nabla}^{k+1} \nabla \cdot\left(\mathbf{u}_{\star}^{k} n^{k}\right) .
\end{aligned}
$$

Substituting (4.20) into (4.19) yields

$$
\begin{aligned}
\frac{f_{\nabla}^{k+1}-f_{\nabla}^{k}}{\delta t_{k}} \leq & \gamma_{\nabla}^{k} \frac{T^{k+1}-T^{k}}{\delta t_{k}}-\mu_{\nabla}^{k+1} \nabla \cdot\left(\mathbf{u}_{\star}^{k} n^{k}\right) \\
& -\nabla \cdot\left(\left(\nabla \cdot\left(\mathbf{u}_{\star}^{k} n^{k}\right)\right) c^{k+1} \nabla n^{k+1}\right) .
\end{aligned}
$$

Finally, (4.17) is a result of summing (4.18) and (4.21).

TheOREM 4.2. Assume that the condition (4.4) holds. The semi-implicit scheme given by (4.5)-(4.9) satisfies the second law of thermodynamics as

$$
\begin{gathered}
\frac{\mathcal{S}^{k+1}-\mathcal{S}^{k}}{\delta t_{k}}+\int_{\partial \Omega} \frac{\mathbf{q}_{\partial \Omega}^{k+1} \cdot \boldsymbol{\nu}_{\partial \Omega}}{T^{k+1}} d s \geq\left\|\frac{\sqrt{\Theta^{k+1}}}{T^{k+1}} \nabla T^{k+1}\right\|^{2}+\left(\eta^{k} D\left(\mathbf{u}^{k+1}\right): \nabla \mathbf{u}^{k+1}, \frac{1}{T^{k+1}}\right) \\
\quad+\left(\lambda^{k}\left|\nabla \cdot \mathbf{u}^{k+1}\right|^{2}+\frac{1}{2 \delta t_{k}} \rho^{k}\left(\left|\mathbf{u}^{k+1}-\mathbf{u}_{\star}^{k}\right|^{2}+\left|\mathbf{u}_{\star}^{k}-\mathbf{u}^{k}\right|^{2}\right), \frac{1}{T^{k+1}}\right) \geq 0
\end{gathered}
$$

where $\mathbf{q}_{\partial \Omega}^{k+1}$ denotes the heat transfer flux between the system and its environment at the time $t^{k+1}$. 
Proof. Since $s=\frac{1}{T}(\vartheta-f)$, we obtain

$$
\begin{aligned}
\frac{\mathcal{S}^{k+1}-\mathcal{S}^{k}}{\delta t_{k}} & =\left(\frac{s^{k+1}-s^{k}}{\delta t_{k}}, 1\right) \\
& =\left(\frac{\vartheta^{k+1}-\vartheta^{k}-f^{k+1}+f^{k}}{\delta t_{k}}, \frac{1}{T^{k+1}}\right)-\left(\frac{T^{k+1}-T^{k}}{\delta t_{k}}, \frac{s^{k}}{T^{k+1}}\right) .
\end{aligned}
$$

Taking into account the relation $s^{k}=-\gamma^{k}$, we substitute (4.17) into (4.23) and obtain

$$
\begin{aligned}
\frac{\mathcal{S}^{k+1}-\mathcal{S}^{k}}{\delta t_{k}} \geq & \left(\frac{\vartheta^{k+1}-\vartheta^{k}}{\delta t_{k}}, \frac{1}{T^{k+1}}\right)+\left(\mu^{k+1} \nabla \cdot\left(n^{k} \mathbf{u}_{\star}^{k}\right), \frac{1}{T^{k+1}}\right) \\
& +\left(\nabla \cdot\left(\left(\nabla \cdot\left(\mathbf{u}_{\star}^{k} n^{k}\right)\right) c^{k+1} \nabla n^{k+1}\right), \frac{1}{T^{k+1}}\right) .
\end{aligned}
$$

It can be obtained from (4.10) that

$$
\begin{aligned}
\left(\frac{\vartheta^{k+1}-\vartheta^{k}}{\delta t_{k}}, \frac{1}{T^{k+1}}\right)= & -\left(\nabla \cdot \mathbf{q}^{k+1}, \frac{1}{T^{k+1}}\right) \\
& -\left(\nabla \cdot\left(\left(\nabla \cdot\left(\mathbf{u}_{\star}^{k} n^{k}\right)\right) c^{k+1} \nabla n^{k+1}\right), \frac{1}{T^{k+1}}\right) \\
& -\left(\mu^{k+1} \nabla \cdot\left(\mathbf{u}_{\star}^{k} n^{k}\right), \frac{1}{T^{k+1}}\right) \\
& +\left(\lambda^{k}\left|\nabla \cdot \mathbf{u}^{k+1}\right|^{2}+\eta^{k} D\left(\mathbf{u}^{k+1}\right): \nabla \mathbf{u}^{k+1}, \frac{1}{T^{k+1}}\right) \\
& +\left(\frac{1}{2 \delta t_{k}} \rho^{k}\left(\left|\mathbf{u}^{k+1}-\mathbf{u}_{\star}^{k}\right|^{2}+\left|\mathbf{u}_{\star}^{k}-\mathbf{u}^{k}\right|^{2}\right), \frac{1}{T^{k+1}}\right) .
\end{aligned}
$$

Substituting (4.25) into (4.24), and taking into account

$$
-\left(\nabla \cdot \mathbf{q}^{k+1}, \frac{1}{T^{k+1}}\right)=-\int_{\partial \Omega} \frac{\mathbf{q}_{\partial \Omega}^{k+1} \cdot \boldsymbol{\nu}_{\partial \Omega}}{T^{k+1}} d s+\left\|\frac{\sqrt{\Theta^{k+1}}}{T^{k+1}} \nabla T^{k+1}\right\|^{2},
$$

we obtain 4.22).

Although the nonlinear coupling relationship between molar density, temperature and velocity has been alleviated to a great extent by a series of semi-implicit treatments, the above time-discrete system still suffers from weakly nonlinear coupling. To solve the discrete systems efficiently, with the help of the auxiliary velocity, we propose the following fully decoupled, linearized iterative method:

$$
\begin{gathered}
\mathbf{u}_{\star}^{k, l}=\mathbf{u}^{k}-\frac{\delta t_{k}}{\rho^{k}}\left(n^{k} \nabla \mu^{k+1, l+1}+s^{k} \nabla T^{k+1, l}\right), \\
\frac{n^{k+1, l+1}-n^{k}}{\delta t_{k}}+\nabla \cdot\left(n^{k} \mathbf{u}_{\star}^{k, l}\right)=0, \\
\rho^{k}\left(\frac{\mathbf{u}^{k+1, l+1}-\mathbf{u}^{k}}{\delta t_{k}}+\mathbf{u}_{\star}^{k, l} \cdot \nabla \mathbf{u}^{k+1, l+1}\right)=-n^{k} \nabla \mu^{k+1, l+1}+\gamma^{k} \nabla T^{k, l}
\end{gathered}
$$




$$
\begin{aligned}
& +\nabla \cdot \eta^{k} D\left(\mathbf{u}^{k+1, l+1}\right)+\nabla\left(\lambda^{k} \nabla \cdot \mathbf{u}^{k+1, l+1}\right), \\
& \frac{\vartheta^{k+1, l+1}-\vartheta^{k}}{\delta t_{k}}+\nabla \cdot\left(\mathbf{u}_{\star}^{k, l} s^{k} T^{k+1, l}\right) \\
& =\nabla \cdot \Theta^{k} \nabla T^{k+1, l+1}-\nabla \cdot\left(\left(\nabla \cdot\left(\mathbf{u}_{\star}^{k, l} n^{k}\right)\right) c^{k+1, l} \nabla n^{k+1, l+1}\right) \\
& -\mu^{k+1, l+1} \nabla \cdot\left(\mathbf{u}_{\star}^{k, l} n^{k}\right)-\mathbf{u}_{\star}^{k, l} \cdot \gamma^{k} \nabla T^{k, l}+\eta^{k} D\left(\mathbf{u}^{k+1, l+1}\right): \nabla \mathbf{u}^{k+1, l+1} \\
& +\lambda^{k}\left|\nabla \cdot \mathbf{u}^{k+1, l+1}\right|^{2}+\frac{1}{2 \delta t_{k}} \rho^{k}\left(\left|\mathbf{u}^{k+1, l+1}-\mathbf{u}_{\star}^{k, l}\right|^{2}+\left|\mathbf{u}_{\star}^{k, l}-\mathbf{u}^{k}\right|^{2}\right),
\end{aligned}
$$

where the superscripts $l$ and $l+1$ denote the $l$ th and $(l+1)$ th iterations respectively and $\mu^{k+1, l+1}, \vartheta^{k+1, l+1}$ are defined as

$$
\begin{aligned}
\mu^{k+1, l+1}= & \mu_{b}^{\text {convex }}\left(n^{k+1, l}, T^{k+1, l}\right)+\frac{\partial \mu_{b}^{\text {convex }}}{\partial n}\left(n^{k+1, l}, T^{k+1, l}\right)\left(n^{k+1, l+1}-n^{k+1, l}\right) \\
& +\mu_{b}^{\text {concave }}\left(n^{k}, T^{k+1, l}\right)+\mu_{\nabla}\left(n^{k+1, l+1}, T^{k+1, l}\right) \\
\vartheta^{k+1, l+1}= & \vartheta\left(n^{k+1, l+1}, T^{k+1, l}\right)+\frac{\partial \vartheta}{\partial T}\left(n^{k+1, l+1}, T^{k+1, l}\right)\left(T^{k+1, l+1}-T^{k+1, l}\right)(4.32)
\end{aligned}
$$

For the above iterative method, using the similar techniques in the proof of Theorem 4.1. we can prove the following theorem.

THEOREM 4.3. The iterative method given by (4.27)-(4.32) satisfies the first law of thermodynamics as

$$
\frac{\mathcal{E}^{k+1, l+1}-\mathcal{E}^{k}}{\delta t_{k}}=-\int_{\partial \Omega} \mathbf{q}_{\partial \Omega}^{k+1} \cdot \boldsymbol{\nu}_{\partial \Omega} d \boldsymbol{s}
$$

where

$$
\begin{gathered}
\mathcal{E}^{k+1, l+1}=\mathcal{H}^{k+1, l+1}+\mathcal{U}^{k+1, l+1} \\
\mathcal{H}^{k+1, l+1}=\frac{1}{2} \int_{\Omega} \rho^{k+1, l+1}\left|\mathbf{u}^{k+1, l+1}\right|^{2} d \mathbf{x}, \quad \mathcal{U}^{k+1, l+1}=\int_{\Omega} \vartheta^{k+1, l+1} d \mathbf{x} .
\end{gathered}
$$

Thanks to the feature that this iterative method satisfies the first law of thermodynamics, it converges rapidly in practical applications.

5. Numerical results. In this section, we employ the proposed method to carry out a series of numerical tests. The simulated substance is n-butane $\left(\mathrm{nC}_{4}\right)$, and its physical data is listed in Table 5.1 in which $\vartheta_{0}, T_{0}$ and $P_{0}$ take the values suggested in [27. The correlation coefficients for the molar heat capacity in (2.1) are taken as 24,27 ]

$$
\alpha_{0}=9.487, \alpha_{1}=3.313 \times 10^{-1}, \alpha_{2}=-1.108 \times 10^{-4}, \alpha_{3}=-2.822 \times 10^{-9} .
$$

The heat conduction coefficient is set to be a constant as $\Theta=0.1 \mathrm{~W} / \mathrm{m} / \mathrm{K}$. The volumetric viscosity and the shear viscosity are taken as $\xi=\eta=10^{-4} \mathrm{~Pa} \cdot \mathrm{s}$. In all numerical tests, to initialize the molar density distributions, we use the following gas and liquid molar densities, denoted by

$$
n_{G}=358.2996 \mathrm{~mol} / \mathrm{m}^{3}, \quad n_{L}=9058.3724 \mathrm{~mol} / \mathrm{m}^{3} .
$$


We use the rectangular domains, and denote the spatial coordinate $\mathbf{x}=(x, y) \in$ $\mathbb{R}^{2}$. The cell-centered finite difference method and the upwind scheme are employed to discretize the mass balance equation and energy balance equation, while the finite volume method on the staggered mesh [29] is used for the momentum balance equation. These spatial discretization schemes can be equivalent to special mixed finite element methods with quadrature rules [2, 11]. The stop criterion of the iterative method for solving the discrete equations is that the 2-norm of the relative variation of molar density, velocity and temperature between the current and previous iterations is less than $10^{-3}$, and the maximum nonlinear iterations are also set to be not larger than 10 for preventing too many loops. These settings are enough to ensure the convergence of nonlinear iterations in the most cases.

Table 5.1: Physical parameters of $\mathrm{nC}_{4}$

\begin{tabular}{ccccccc}
\hline$M_{w}(\mathrm{~g} / \mathrm{mol})$ & $P_{c}(\mathrm{bar})$ & $T_{c}(\mathrm{~K})$ & $\omega$ & $\vartheta_{0}(\mathrm{~J} / \mathrm{mol})$ & $T_{0}(\mathrm{~K})$ & $P_{0}(\mathrm{bar})$ \\
\hline 58.12 & 38.0 & 425.2 & 0.199 & -2478.95687512 & 298.15 & 1 \\
\hline
\end{tabular}

5.1. Isolated system. In this example, we consider an ideal isolated system, which exchanges no mass or heat energy with its environment. The computational domain is a square as $\Omega=(-L, L)^{2}$, where $L=10 \mathrm{~nm}$, and a uniform rectangular mesh with $40 \times 40$ elements is applied. We take a fixed time step size $\delta t=3 \times 10^{-13} \mathrm{~s}$, and simulate the dynamics of this system for 500 time steps. The initial temperature of this system is homogeneous and equal to $345 \mathrm{~K}$. The initial molar density is defined by the following function

$$
n=\left\{\begin{array}{l}
n_{L}, \quad|x| \leq r \text { and }|y| \leq r, \\
n_{G}, \quad \text { elsewhere }
\end{array}\right.
$$

where $r=0.35 L$. Namely, a square droplet is initially located at the center of the domain. The discrete initial molar density is also illustrated in Figure 5.1(a) The initial velocity is zero. In the simulation time, we always set the boundary conditions $\mathbf{q} \cdot \boldsymbol{\nu}_{\Omega}=0$ on the boundary $\partial \Omega$.

In Figures 5.1, we illustrate the molar density profiles at different time steps, while the temperature profiles and velocity fields at different time steps are depicted in Figures 5.2 and Figures 5.3 respectively.

It is obviously observed from Figures 5.1 that the droplet changes from a square to a circle due to the effect of the interfacial tension. Figures 5.2 show that the region around the droplet has higher temperatures than the gas region, but the temperature fields tend towards a homogeneous distribution during the evolution of this system. The velocity fields in Figures 5.3 depict the flow evolutions with the mass and temperature variations; in particular, we can see that the magnitudes of both velocity components decrease with time steps especially after the 50th time step. From these results, it can be predicted that the system will tend towards a equilibrium state infinitely.

5.2. Bubble dropping under a boundary temperature contrast. In this example, we consider a square domain $\Omega=(-L, L)^{2}$, where $L=10 \mathrm{~nm}$, and we use a uniform rectangular mesh with $40 \times 40$ elements. The initial molar density is distributed by the following function

$$
n=\frac{1}{2}\left(n_{L}+n_{G}\right)+\frac{1}{2}\left(n_{L}-n_{G}\right) \tanh (w(d-r) / L)
$$




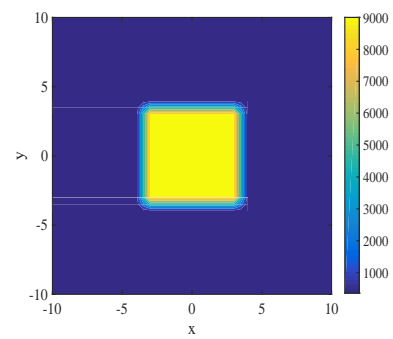

(a)

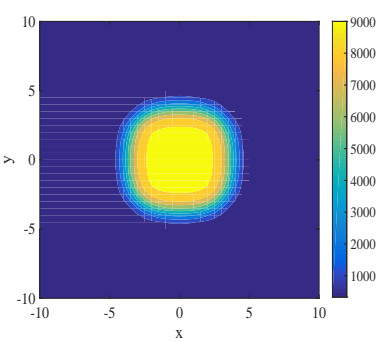

(b)

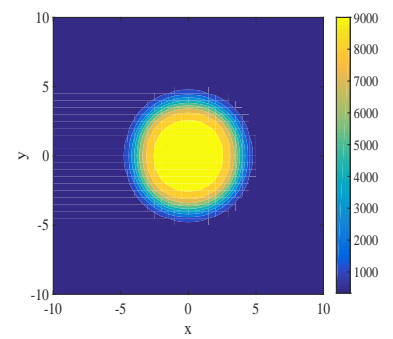

(c)

Fig. 5.1: Dynamics of an isolated system: the initial molar density distribution(a) and the molar density distributions at the 200th(b) and 500th(c) time step respectively.

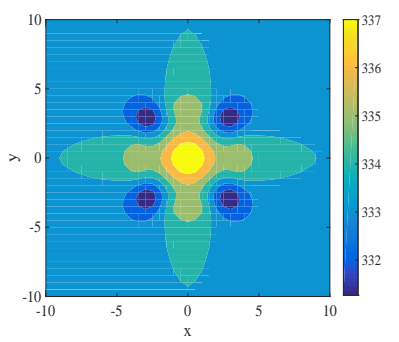

(a)

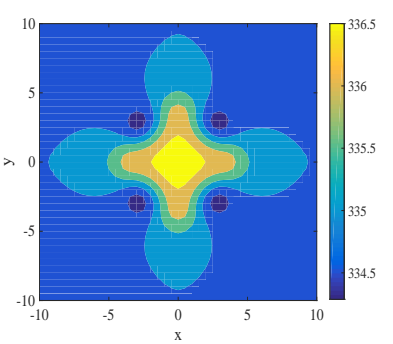

(b)

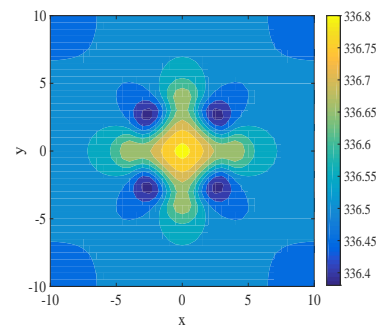

(c)

Fig. 5.2: Dynamics of an isolated system: the temperature profiles at the 50th(a), 200th(b) and 500th(c) time step respectively.

where $w=10^{5}, r=0.45 L$ and $d=\sqrt{x^{2}+y^{2}}$. The discrete distribution of the initial molar density is illustrated in Figure $5.4(\mathrm{a})$.

We partition the domain boundary $\partial \Omega$ into three non-overlapping subdivisions as

$$
\partial \Omega=\Gamma_{n} \cup \Gamma_{t} \cup \Gamma_{b},
$$

where

$$
\begin{gathered}
\Gamma_{n}=\left\{\partial \Omega \cap\left\{\mathbf{x} \in \mathbb{R}^{2} \mid x=-L\right\}\right\} \cup\left\{\partial \Omega \cap\left\{\mathbf{x} \in \mathbb{R}^{2} \mid x=L\right\}\right\}, \\
\Gamma_{t}=\partial \Omega \cap\left\{\mathbf{x} \in \mathbb{R}^{2} \mid y=L\right\}, \\
\Gamma_{b}=\partial \Omega \cap\left\{\mathbf{x} \in \mathbb{R}^{2} \mid y=-L\right\} .
\end{gathered}
$$

The initial temperature is uniformly equal to $345 \mathrm{~K}$ inside $\Omega$, and the boundary conditions are imposed through the simulation time,

$$
T=345 \mathrm{~K} \text { on } \Gamma_{t}, \quad T=348 \mathrm{~K} \text { on } \Gamma_{b}, \quad \mathbf{q} \cdot \boldsymbol{\nu}_{\Omega}=0 \text { on } \Gamma_{n} .
$$

Namely, there exists a temperature contrast between the top and bottom of this domain. The initial velocity is fixed to be zero.

We take a fixed time step size $\delta t=5 \times 10^{-13} \mathrm{~s}$, and use 50000 time steps to simulate this problem. 


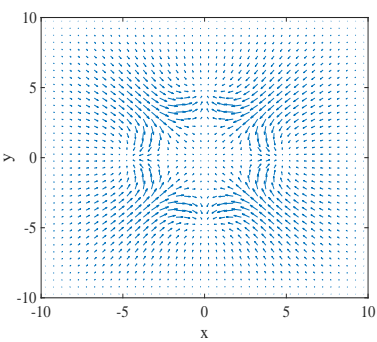

(a)

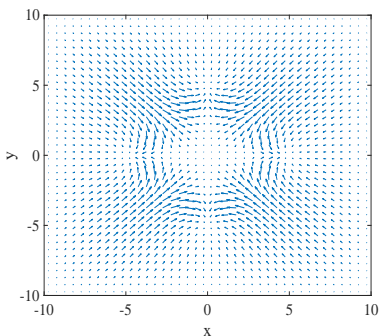

(d)

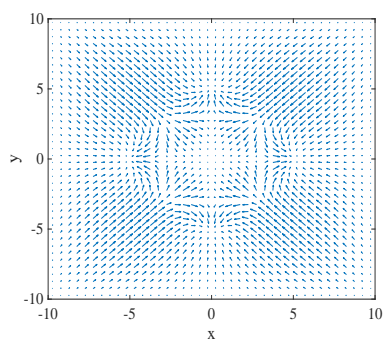

(g)

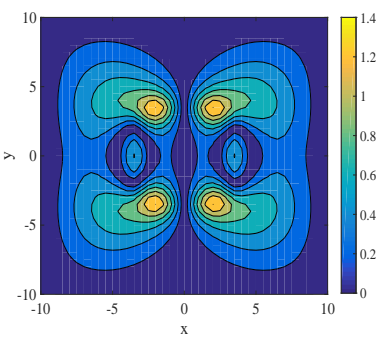

(b)

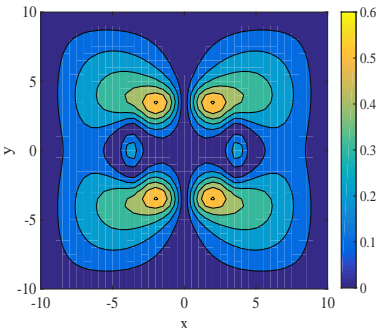

(e)

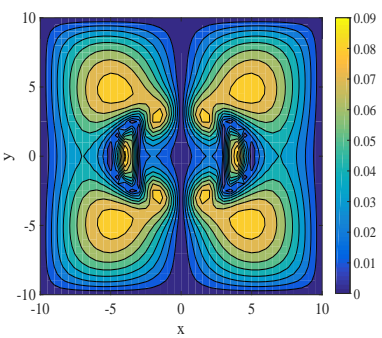

(h)

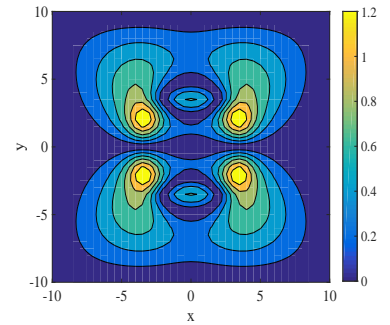

(c)

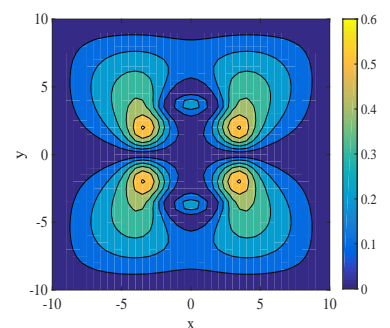

(f)

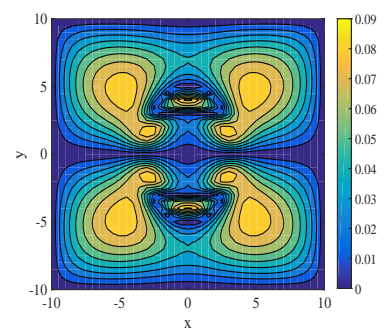

(i)

Fig. 5.3: Dynamics of an isolated system: the flow quivers (left column), magnitude contours of x-direction velocity component (center column), and magnitude contours of y-direction velocity component (right column) at the 50th(top row), 200th(center row), and 500th(bottom row) time step respectively.

In Figures 5.4, we illustrate the molar density profiles at various time steps. In Figures [5.5 the temperature profiles are depicted at various time steps. In Figures [5.6. we illustrate the velocity fields, especially magnitudes of both velocity components at different time steps.

From Figures 5.4, we can see that the initial bubble with a rough shape becomes a smooth circle, and it is gradually dropping towards the bottom. Figures 5.5 show that there exists a temperature contrast between the top and bottom of this domain, although the temperature fields vary due to the bubble motion. Figures 5.6 depict that the velocity fields are generated by the temperature contrast and the fluid flows towards the bottom. Finally, the system will reach a steady state; i.e., the bubble spreads into a semicircle on the heated bottom, the velocity field vanishes and the temperature has the layered distribution along the y-direction.

6. Conclusions. We have studied modeling and numerical simulation of a diffuseinterface model of gas-liquid two-phase flow in an inhomogeneous temperature field. 


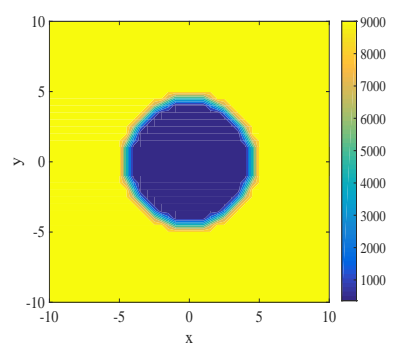

(a)

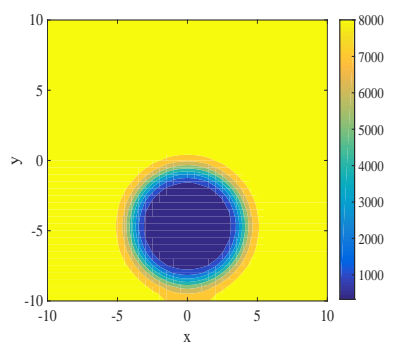

(d)

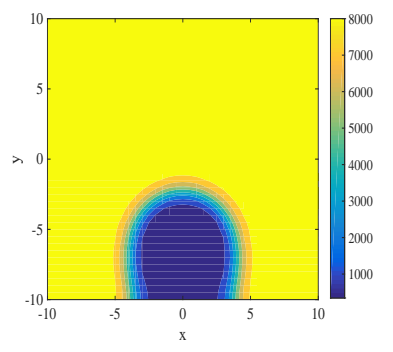

(g)

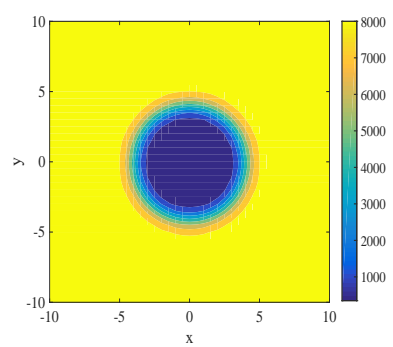

(b)

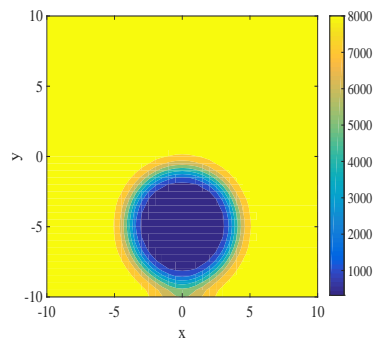

(e)

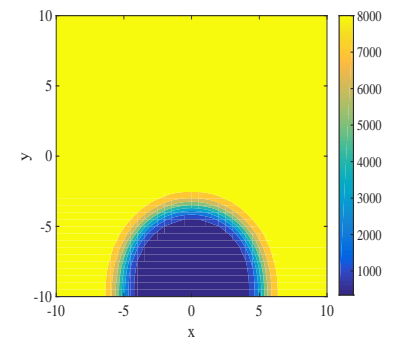

(h)

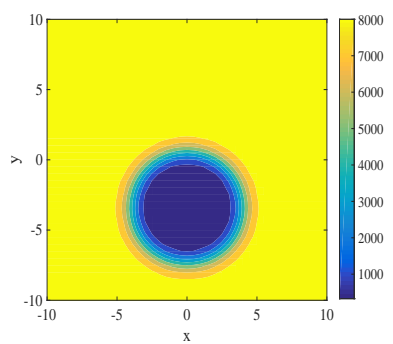

(c)

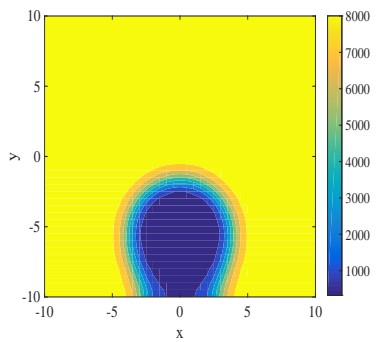

(f)

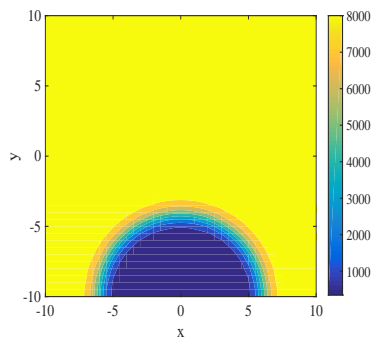

(i)

Fig. 5.4: Bubble dropping problem: the molar density profiles at the initial time(a), 1000th(b), 20000th(c), 28000th(d), 29000th(e), 30000th(f), 30500th(g), 32000th(h) and 50000 th(i) time step respectively.

It is different from the existing models that we employ the Peng-Robinson equation of state instead of the van der Waals equation of state, and use a realistic temperaturedependent influence parameter in the gradient contribution of Helmholtz free energy density. As a result, this model is capable of describing physical behaviors of numerous realistic gas-liquid fluids accurately, such as $\mathrm{N}_{2}, \mathrm{CO}_{2}$ and hydrocarbons.

In order to resolve the difficulty resulting from the complicate form of thermodynamical pressure, we prove a relation associating the pressure gradient with the gradients of temperature and chemical potential. Using this relation, we reformulate the model equations, which is beneficial to theoretical analysis and numerical simulation. The new formulation of momentum equation shows that chemical potential and temperature gradients become the primary driving force of the fluid motion. By the new formulations, we prove that the model obey the first and second laws of thermodynamics.

To design efficient numerical time schemes, we prove that the bulk contribution of Helmholtz free energy density is a concave function with respect to the temperature 


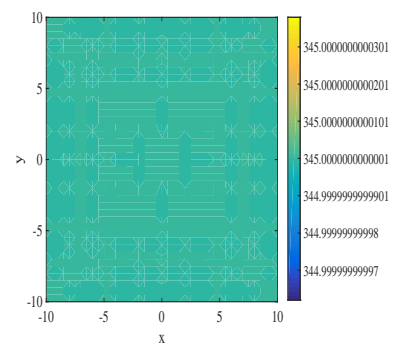

(a)

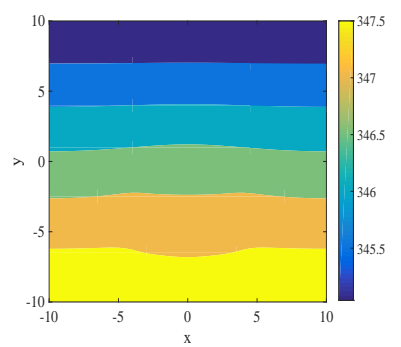

(d)

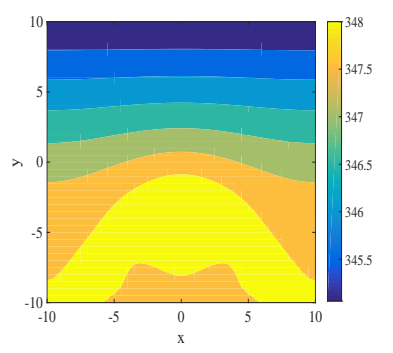

(g)

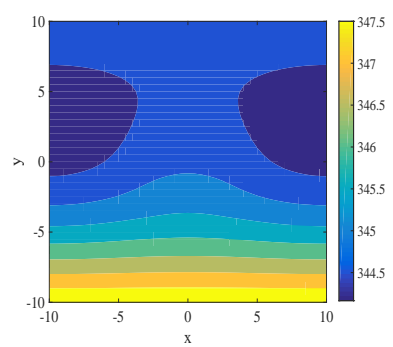

(b)

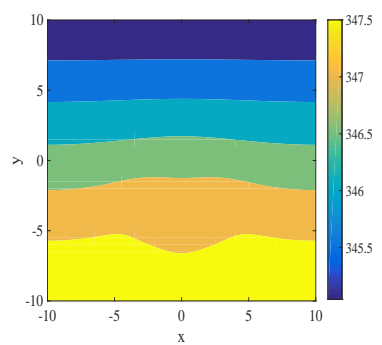

(e)

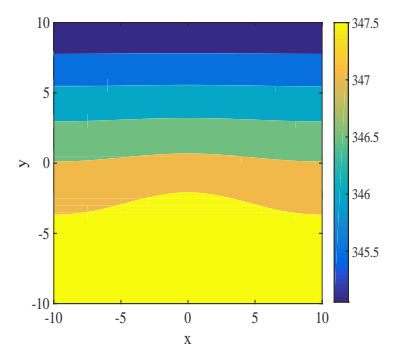

(h)

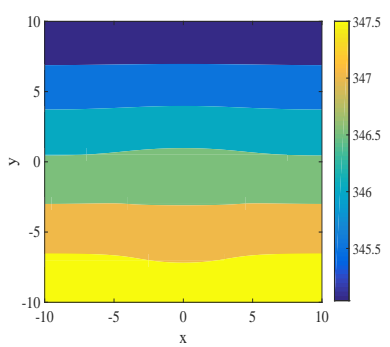

(c)

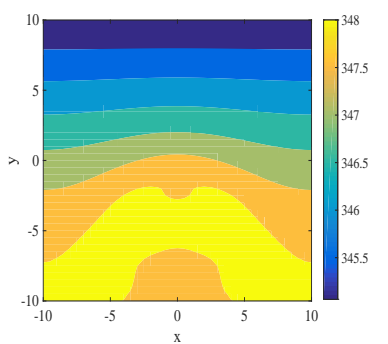

(f)

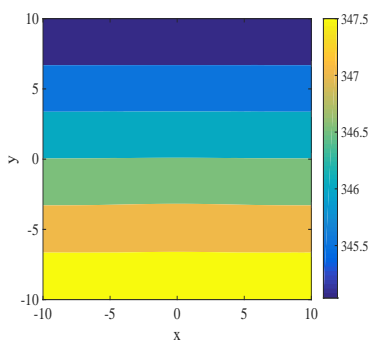

(i)

Fig. 5.5: Bubble dropping problem: the temperature profiles at the initial time(a), 1000th(b), 20000th(c), 28000th(d), 29000th(e), 30000th(f), 30500th(g), 32000th(h) and 50000 th(i) time step respectively.

and its gradient contribution is also concave with respect to the temperature under certain conditions. Based on the proposed modeling formulations, we propose a novel thermodynamically consistent numerical scheme by applying the convex-concave splitting of Helmholtz free energy density. The proposed scheme also utilizes an auxiliary velocity, which depends on molar density and temperature, to alleviate the nonlinear coupling relation between molar density, velocity and temperature. Furthermore, a decoupled, linearized iterative method is developed for solving the discrete equations. It is also proved with a mathematical rigor that the proposed time-marching scheme satisfies the first and second laws of thermodynamics. Using the proposed numerical method, we have carried out a series of numerical tests and investigate the simulation results.

\section{REFERENCES}

[1] H. Abels, H. Garcke and G. Grün. Thermodynamically consistent, frame indifferent diffuse 
interface models for incompressible two-phase flows with different densities. Mathematical Models and Methods in Applied Sciences, Vol. 22, No. 3, 1150013, 2012.

[2] T. Arbogast, M.F. Wheeler, and I. Yotov. Mixed finite elements for elliptic problems with tensor coefficients as cell-centered finite differences. SIAM Journal on Numerical Analysis, pages 828-852, 1997.

[3] K. Bao, Y. Shi, S. Sun, and X.-P. Wang. A finite element method for the numerical solution of the coupled Cahn-Hilliard and Navier-Stokes system for moving contact line problems. Journal of Computational Physics, 231(24): 8083-8099, 2012.

[4] J. Bueno, H. Gomez. Liquid-vapor transformations with surfactants. Phase-field model and Isogeometric Analysis. Journal of Computational Physics, 321: 797-818, 2016.

[5] J. W. Cahn, J. E. Hilliard. Free Energy of a Nonuniform System. I. Interfacial Free Energy. Journal of Chemical Physics, 28: 258-267, 1958.

[6] Z. Chen, G. Huan, Y. Ma, Computational methods for multiphase flows in porous media. SIAM Comp. Sci. Eng., Philadelphia, 2006.

[7] Y. Chen, J. Shen. Efficient, adaptive energy stable schemes for the incompressible Cahn-Hilliard Navier-Stokes phase-field models. Journal of Computational Physics, 308: 40-56, 2016.

[8] S. R. De Groot, and P. Mazur. Non-Equilibrium Thermodynamics. Dover Publications, New York, 2011.

[9] A. Firoozabadi. Thermodynamics of hydrocarbon reservoirs. McGraw-Hill New York, 1999.

[10] X. Fan, J. Kou, Z. Qiao, and S. Sun. A Componentwise Convex Splitting Scheme for Diffuse Interface Models with Van der Waals and Peng-Robinson Equations of State. SIAM Journal on Scientific Computing, 39(1): B1-B28, 2017.

[11] V. Girault, H. Lopez. Finite-element error estimates for the MAC scheme. IMA Journal of Numerical Analysis, 16(3): 347-379, 1996.

[12] T. Jindrová and J. Mikyška. General algorithm for multiphase equilibria calculation at given volume, temperature, and moles. Fluid Phase Equilibria, 393:7-25, 2015.

[13] J. Kou, S. Sun, and X. Wang. Efficient numerical methods for simulating surface tension of multi-component mixtures with the gradient theory of fluid interfaces. Computer Methods in Applied Mechanics and Engineering, 292: 92-106, 2015.

[14] J. Kou and S. Sun. Numerical methods for a multi-component two-phase interface model with geometric mean influence parameters. SIAM Journal on Scientific Computing, 37(4): B543-B569, 2015.

[15] J. Kou and S. Sun. Unconditionally stable methods for simulating multi-component two-phase interface models with Peng-Robinson equation of state and various boundary conditions. Journal of Computational and Applied Mathematics, 291(1): 158-182, 2016.

[16] J. Kou, S. Sun, and X. Wang. An energy stable evolution method for simulating two-phase equilibria of multi-component fluids at constant moles, volume and temperature. Computational Geosciences, 20: 283-295, 2016.

[17] J. Kou and S. Sun. Multi-scale diffuse interface modeling of multi-component two-phase flow with partial miscibility. Journal of Computational Physics, 318: 349-372, 2016.

[18] J. Kou and S. Sun. Efficient energy-stable dynamic modeling of compositional grading. International Journal of Numerical Analysis and Modeling, 14(2):218-242, 2017.

[19] J. Liu, C. M. Landis, H. Gomez, T. J.R. Hughes. Liquid-vapor phase transition: Thermomechanical theory, entropy stable numerical formulation, and boiling simulations. Computer Methods in Applied Mechanics and Engineering, 297: 476-553, 2015.

[20] C. Miqueu, B. Mendiboure, C. Graciaa and J. Lachaise. Modelling of the surface tension of binary and ternary mixtures with the gradient theory of fluid interfaces. Fluid Phase Equilibria, 218:189-203, 2004.

[21] A. Onuki. Dynamic van der Waals theory of two-phase fluids in heat flow. Physical Review Letters, 94(5): 054501, 2005.

[22] A. Onuki. Dynamic van der Waals theory. Physical Review E, 75(3): 036304, 2007.

[23] D. Peng and D.B. Robinson. A new two-constant equation of state. Industrial and Engineering Chemistry Fundamentals, 15(1):59-64, 1976.

[24] R.C. Reid, J.M. Prausnitz, B.E. Poling. The Properties of Gases and Liquids, fourth ed., McGraw-Hill, New York, USA, 1987.

[25] Z. Qiao and S. Sun. Two-phase fluid simulation using a diffuse interface model with PengRobinson equation of state. SIAM Journal on Scientific Computing, 36(4): B708-B728, 2014 . 
[26] J. Shen, X. Yang. Decoupled, energy stable schemes for phase-field models of two-phase incompressible flows. SIAM Journal on Numerical Analysis, 53(1): 279-296, 2015.

[27] T. Smejkal, J. Mikyška. Phase stability testing and phase equilibrium calculation at specified internal energy, volume, and moles. Fluid Phase Equilibria, 431: 82-96, 2017.

[28] M. T. Taylor, T. Qian. Thermal singularity and contact line motion in pool boiling: Effects of substrate wettability. Physical Review E, 93(3): 033105, 2016.

[29] G. Tryggvason, R. Scardovelli and S. Zaleski. Direct Numerical Simulations of Gas-Liquid Multiphase Flows. Cambridge University Press, New York, 2011. 


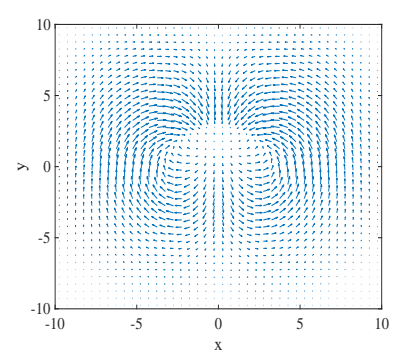

(a)

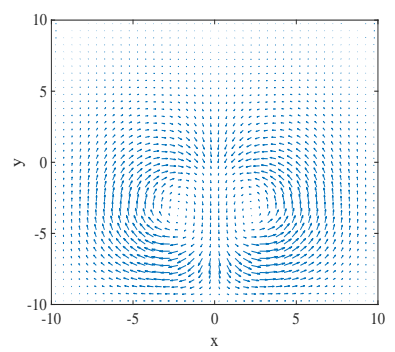

(d)

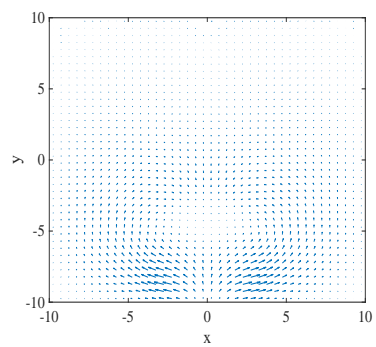

(g)

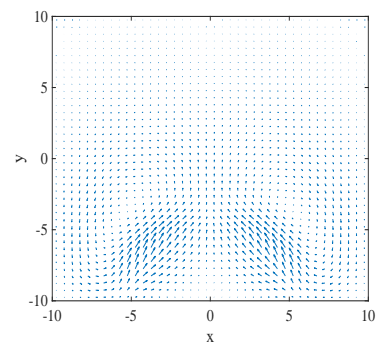

(j)

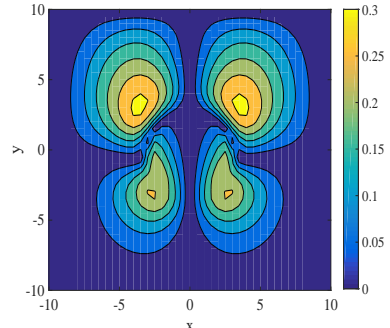

(b)

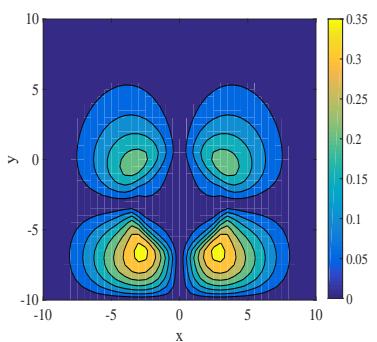

(e)

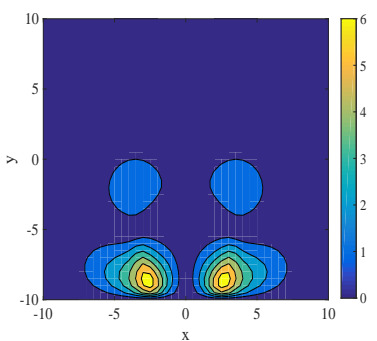

(h)

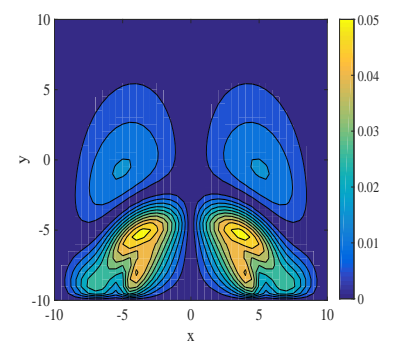

(k)

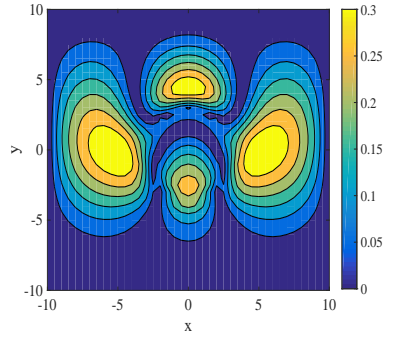

(c)

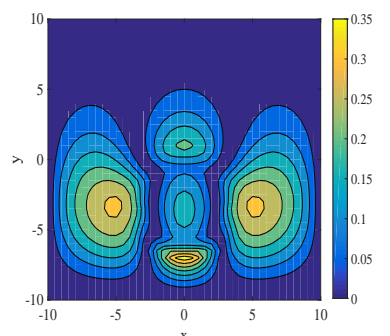

(f)

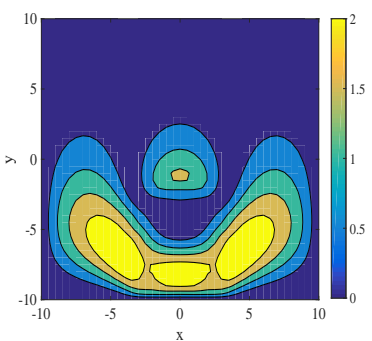

(i)

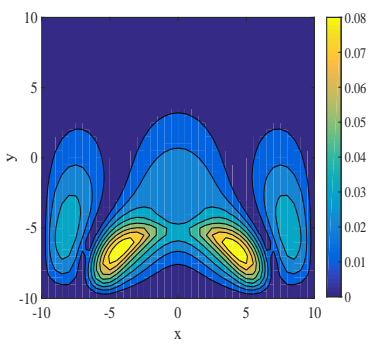

(l)

Fig. 5.6: Bubble dropping problem: the flow quivers (left column), magnitude contours of $\mathrm{x}$-direction velocity component (center column), and magnitude contours of ydirection velocity component (right column) at the 1000th(the first row), 20000th(the second row), 30000th(the third row), and 50000th(the bottom row) time step respectively. 